\title{
Interaction of Synthetic Cannabinoid Receptor Agonists with Cannabinoid Receptor I: Insights into Activation Molecular Mechanism
}

Sergei Gavryushov, ${ }^{1,2 *}$ Anton A. Bashilov, ${ }^{2,3}$ Konstantin V. Cherashev-Tumanov, ${ }^{2}$ Nikolay N. Kuzmich, ${ }^{4,2}$ Tatyana I. Burykina, ${ }^{2}$ and Boris N. Izotov ${ }^{2}$

${ }^{1}$ Engelhardt Institute of Molecular Biology, Russian Academy of Sciences, Vavilova str. 32, Moscow, 119991, Russian Federation

${ }^{2}$ Sechenov First Moscow State Medical University, 2-4 Bolshaya Pirogovskaya str., Moscow, 119991, Russian Federation

${ }^{3}$ Skolkovo Institute of Science and Technology, Skolkovo 143025, Moscow, Russian Federation

${ }^{4}$ Smorodintsev Research Institute of Influenza, WHO National Influenza Centre of Russia, 15/17 Professor Popov str., Saint-Petersburg, 197376, Russian Federation

*corresponding author, email: sergei_gavryushov@yahoo.com 


\begin{abstract}
Synthetic cannabinoid receptor agonists (SCRAs) have become a wide group of new psychoactive substances since 2010s. In contrast to natural endogenous endocannabinoids, these agonists of cannabinoid receptor $\mathrm{I}\left(\mathrm{CB}_{1}\right)$ bear some structural similarity to natural phytocannabinoids such as (-)-trans- $\Delta^{9}$-tetrahydrocannabinol, the principal psychoactive substance of cannabis. Recently X-ray structures of complexes of $\mathrm{CB}_{1}$ with SCRAs as well as complexes of $\mathrm{CB}_{1}$ with its antagonist have been published. Based on those data, we have analyzed SCRA-CB 1 interaction in detail, using molecular modeling and molecular dynamics simulations. The molecular mechanism of conformational transformation of the transmembrane domain of $\mathrm{CB}_{1}$ caused by its interaction with SCRA was studied. These conformational changes allosterically modulate the $\mathrm{CB}_{1}-\mathrm{G}_{i}$ complex, providing activation of $\mathrm{G}_{i}$ protein. Based on an $\mathrm{X}$ ray determined structure of inactive $\mathrm{CB}_{1}$-antagonist complex, there was modeled a conformation of inactive $\mathrm{CB}_{1}$ that overcame some high potential barriers of the structural transformation of $\mathrm{CB}_{1}$ from its inactive conformation in this complex to the active state. For that model molecular dynamics simulations of SCRA binding to $\mathrm{CB}_{1}$ led to the active state of $\mathrm{CB}_{1}$, which allowed us to explore the key features of this activation and the molecular mechanism of the receptor's structural transformation. One of conclusions is a new method to detect the active-state conformation of the class-A G-protein-coupled receptor $\mathrm{CB}_{1}$ different from unreliable recognition of movement of transmembrane helix 6 affected by many factors in crystal complexes.
\end{abstract}

Keywords: cannabinoid receptor I $\bullet$ GPCR activation mechanism $\bullet$ apo receptor model $\bullet$ agonist ligand binding $\bullet \mathrm{MD}$ simulations of $\mathrm{CB} 1$ receptor activation 


\section{Introduction}

Cannabinoid type 1 receptor $\left(\mathrm{CB}_{1}\right)$ is a G-protein-coupled receptor (GPCR) in the central and peripheral nerves systems. ${ }^{1} \mathrm{CB}_{1}$ is one of the most abundant GPCRs in the brain, ${ }^{2}$ where its activation in axon terminals of neurons inhibits neurotransmission via presynaptic mechanisms. ${ }^{3}$ Agonists of $\mathrm{CB}_{1}$ affect cognition, motivation, memory, analgesia and motor function. ${ }^{4}$ Ligands that activate $\mathrm{CB}_{1}$ are divided into structurally distinct endogenous cannabinoids (endocannabinoids) and exogenous ligands. Endocannabinoids are lipid-signaling molecules involved in the natural regulation, whereas exogenous ligands are poorly water-soluble and include compounds such as (-)-trans- $\Delta^{9}$-tetrahydrocannabinol $\left(\Delta^{9}-\mathrm{THC}\right)$ - the principal psychoactive component of cannabis - and numerous synthetic cannabinoid receptor agonists (SCRAs) intended to elicit psychoactive effects. Despite the diversity of the latter compounds, all of them bear common structural features. ${ }^{5}$

Recently crystal structures of $\mathrm{CB}_{1}$ complexes with its agonist ${ }^{6}$ and antagonist ${ }^{7}$ ligands have been obtained using X-ray crystallography. They revealed binding of exogenous agonist ligands in the extracellular area of the GPCR channel (the orthosteric binding pocket). A comparison of $\mathrm{CB}_{1}$ structures with agonist and antagonist ligands bound clearly demonstrates the active and inactive conformations of $\mathrm{CB}_{1}$ indicated by transverse movement of $\alpha$-helix transmembrane 6 (TM6) that allosterically modulates G-protein binding to the GPCR receptor. Further reported crystal structures of $\mathrm{CB}_{1}$ complexes with its agonist ligands included interactions of $\mathrm{CB}_{1}$ with $\mathrm{G}_{i}$ protein. $^{8,9}$

In ref. 8 two structures of complexes of $\mathrm{CB}_{1}$ with its agonists were compared to a structure of $\mathrm{CB}_{1}$-antagonist complex. The analysis was supplemented by molecular dynamics (MD) simulations. Finally, some important conclusions about the receptor activation mechanism were drawn. They include existence of a single active conformation of $\mathrm{CB}_{1}$ for different agonists bound, a crucial role of the stacking interactions of hydrophobic residues of TM2 with ligand, a deep hydrophobic pocket in the protein structure to bind the ligand aliphatic chain, a possible role of the GPCR "toggle switch" for $\mathrm{CB}_{1}$ and a list of receptor's residues interacting with the ligand. ${ }^{8}$ Such unique features of $\mathrm{CB}_{1}$ as absence of strong atom-pair interactions with agonist ligand and their common hydrophobic nature were also mentioned. However, the authors concluded incompleteness of the $\mathrm{CB}_{1}$ activation description.

In ref. 9 the activation processes of $\mathrm{CB}_{1}$ were compared with those for cannabinoid receptor 2 $\left(\mathrm{CB}_{2}\right)$. The study was also supplemented by intense computer MD simulations of the ligand binding. Some new details of conformational rearrangement at the receptor activation were clarified. In particular, there were described important details of interactions between residues of 
TM5 and TM7 $\alpha$-helices as well as those of TM2 and TM6 during the receptor activation. An important suggestion about external binding site of endocannabinoids was discussed. However, despite the authors claim that their results reveal the activation mechanism, the entire process of allosteric modulation caused by the ligand binding is not quite clear yet. It especially concerns the role of particular fragments of exogenous agonists in protein conformational changes at the receptor activation.

Despite numerous SCRAs that have been in the market for the past decade are structurally heterogeneous, they share common structural features and comprise four subunits: head, linker, core, and tail..$^{5}$ The aromatic cores identified are typically indoles or azaindoles, although pyrroles, thiazoles and napthalenes have also been reported. Linkers connect the core and the head. Typical linkers are ketones, amides and esters. Heads can be presented by various cyclic and acyclic functional groups. Their size rarely exceeds $10 \AA$. The tail can be an aliphatic chain sometimes bearing an $\omega$-halogen atom. Such a chain can include a benzene ring. The aliphatic chain length does not exceed seven carbon atoms. More importantly, this chain cannot be shorter than four carbon atoms ${ }^{5}$ since the ligand albeit binds but ceases to activate the $\mathrm{CB}_{1}$ receptor. ${ }^{10-12}$

In the present study we have explored the role of subunits of $\mathrm{CB}_{1}$ exogenous agonists and involvement of the ligand's environment in ligand-receptor interaction. Based on the published crystal structures of $\mathrm{CB}_{1}$ complexes with its agonist and antagonist ligands and using computer molecular modeling and both classic and biased MD simulations techniques, we simulated the process of allosteric modulation in $\mathrm{CB}_{1}$ at agonist ligand binding. In other words, we simulated a structural transformation of the receptor from its inactive state to the active-state conformation. Additionally, modeled ligand modifications and mutations of protein amino acids allowed us to verify the role of ligand subunits and clarify the role of particular amino acid residues in the $\mathrm{CB}_{1}$ activation process.

\section{Methods}

\section{Docking of ligands into receptor and MD simulations}

Protein Preparation Wizard of Schrodinger suite of programs was employed to prepare the protein X-ray structures by adding hydrogen atoms, missing side chains and assigning ionization state for aminoacid residues and ligands at physiological $\mathrm{pH}$ value. The docking calculations were done using Glide XP module of Schrodinger 2017-1 suite of programs. ${ }^{13}$ Receptor grid was generated using a default algorithm and a $10 \AA$ box centered on the receptor's co-crystallized ligand. All the ligands were prepared using LigPrep. ${ }^{14}$ Some of the final placements of ligands 
were completed manually using molecular modeling software. The membrane region around receptor was taken from the Uniprot database. ${ }^{15}$ The following MD simulations were performed using Desmond module of Schrodinger 2017-1 suite of programs ${ }^{16}$ with OPLS3 force field. ${ }^{17}$ For periodic boundary conditions (PBC) simulations the transmembrane domain of $\mathrm{CB}_{1}$ was placed into rectangular box with buffer size of $25 \AA$. The POPE $(310 \mathrm{~K})$ membrane model was applied. The protein charges were neutralized by adding $0.15 \mathrm{M} \mathrm{NaCl}$ with excess of chloride ions into TIP3P water. The equilibration of the system was achieved by consequent simulations of brownian dynamics $(10 \mathrm{~K})$ and consequent molecular dynamics (NVT, 10K; NPT, 10K; NPT, $310 \mathrm{~K})$. The final productive MD runs were performed at $310 \mathrm{~K}$ in NPT ensemble. When necessary (as described in the Results section), the restraining potential (force constant 80 $\mathrm{kcal} / \AA^{2} / \mathrm{mol}$ ) was applied to nitrogen and carbon atoms of the protein backbone as well as to heavy atoms of ligands.

\section{Alchemical free energy calculations}

The ddG calculations were performed using FEP+ module (Desmond 5.8) of Schrodinger 2019-2 suite of programs. ${ }^{18-20}$ The biologically irrelevant fragments were removed from 5XRA and 5TGZ structures which were prepared using Protein Preparation Wizard. ${ }^{21}$ The termini were capped, bond orders were assigned, hydrogens were added and missing amino acid side chains were filled with Prime. ${ }^{21-22}$ The titratable residues protonation states were visually inspected and the complex underwent minimization (RMSD=0.5A) using OPLS3e forcefield ${ }^{23}$ which was also used by all subsequent simulations. Since the ligand nitrate group in 5TGZ complex has not been structurally resolved, the standard precision (SP) Glide docking ${ }^{24}$ with core constraint was used for placement of the complete ligand. All the ligands were prepared using LigPrep ${ }^{25}$ and the missing torsion parameters were fit with ForceField Builder. For PBC simulation the complexes were neutralized with $\mathrm{Cl}$ - ions embedded in POPC lipid pre-aligned membrane according to the Orientations of Proteins in Membranes (OPM) database ${ }^{26}$ and placed into SPC water box with buffer sizes of 20,20, and $15 \AA$. The system was minimized by 500 ps of Brownian dynamics keeping the protein and ligand heavy atoms restrained $\left(10 \mathrm{kcal} / \AA^{2} / \mathrm{mol}\right)$ and then relaxed using the default membrane relaxation protocol. The end temperature was $310 \mathrm{~K}$. The ligands before FEP+ calculation were aligned to the native one (by maximum common substructure) and perturbation maps with optimal topology have been constructed. Every perturbation consisted of 12 lambda windows $20 \mathrm{~ns}$ long each in $\mu \mathrm{VT}$ ensemble for both ligand and amino acid transformations. Replica exchange with solute tempering (REST), an enhanced sampling technique introduced by Wang et al. ${ }^{27}$ was employed. By default, the REST region atoms were 
the mutated ones. Also grand canonical Monte Carlo (GCMC) ${ }^{28}$ method was used to enhance sampling of water molecules in the binding site. Analysis of selected trajectory frames was performed using reanalyze_fep.py script when necessary. For every edge of the perturbation map the ddG values were computed using Multistate Bennett Acceptance Ratio (MBAR) method and were given both with and without cycle closure correction (CCC).

\section{Metadynamics simulations}

They were also performed using Desmond 5.8 of Schrodinger 2019-2 suite. The ligand was removed from PDB 5TGZ complex and the system was built and relaxed as described above. The further staged relaxation was carried out at $310 \mathrm{~K}$ in NPT ensemble and included $5 \mathrm{~ns}$ simulation with protein heavy atoms restrained $\left(10 \mathrm{kcal} / \AA^{2} / \mathrm{mol}\right)$, then $1 \mathrm{~ns}$ keeping protein backbone atoms restrained $\left(5 \mathrm{kcal} / \AA^{2} / \mathrm{mol}\right)$ followed by $10 \mathrm{~ns}$ simulation of unrestrained protein. We used Nosé-Hoover thermostat with relaxation time $1.0 \mathrm{ps}$ and Martyna-Tobias-Klein barostat with relaxation time $2.0 \mathrm{ps}$ for temperature and pressure control, respectively. The relaxed $\mathrm{CB}_{1}$ inactive apo conformation was aligned to $\mathrm{CB}_{1}$ molecule in the active state (5XRA) having amino acid residues 195-199, 243-249 and 275-289 as anchor points. We chose two collective variables (CV) describing the transformation: the distance between C-alpha atoms of F381 and V179 (CV1) and the angle between $\mathrm{C}$-alpha atoms of T125, P113 and F102 residues (CV2). Decrease of CV1 characterized the approaching of TM3 to its position in the $\mathrm{CB}_{1}$ 's active-state conformation while increase of $\mathrm{CV} 2$ elevated resolved part of $\mathrm{N}$-end loop, removing it from the path of V179 move and facilitating helix shift. CV1 values were changing to $13 \AA$, approximately corresponding to the CV1 value of 5XRA structure and CV2 values increasing from 55.2 degrees. The simulations themselves were run at $310 \mathrm{~K}$ in NPT ensemble for $100 \mathrm{~ns}$ with the same thermostat and barostate as stated above. Height of Gaussian repulsive potential was set to $0.03 \mathrm{kcal} / \mathrm{mol}$, time interval for addition of Gaussian repulsive potential was $0.09 \mathrm{ps}$, RMS width of the Gaussian repulsive potential was $0.05 \AA$ for CV1 and 2.5 degrees for CV2. The frames were recorded every $10 \mathrm{ps}$. One of the frames corresponding to PES minimum was checked for stability by $500 \mathrm{~ns}$ conventional MD run at $310 \mathrm{~K}$ in NPT ensemble. 


\section{Results}

\section{Docking of different $\mathrm{CB}_{1}$ ligands into the receptor in its active-state and inactive-state conformations}

The previously published crystal structures of active ${ }^{6}$ and inactive ${ }^{7}$ conformations of $\mathrm{CB}_{1}$ allow us to $(i)$ inquire whether the active state is unique for different and diverse agonists bound and (ii) compare the two conformations to find out the key structural changes. The first question was raised in ref. 8, where it was suggested that the receptor adopted two discrete states: active and inactive one. A necessary condition of the active structure uniqueness is a possibility to dock any known agonist ligand into the receptor's active conformation and verify its stable binding to the protein molecule (at least for exogenous ligands). As will be shown, an analysis of docking of antagonist ligands into the $\mathrm{CB}_{1}$ molecule can improve our understanding of the conformation changes between the inactive and active states of the receptor.

For computer molecular modeling of docking of ligands into the molecule of $\mathrm{CB}_{1}$, nine agonist ligands were selected. ${ }^{5,29-40}$ A list of them is given in Table $\mathbf{1}$ along with five inverse agonist (antagonist) ligands ${ }^{41-45}$ also selected for docking.

TABLE 1: List of ligands of $\mathrm{CB}_{1}$ chosen for docking

\begin{tabular}{|c|c|c|c|}
\hline Name & Structure & Type & Function \\
\hline AM- $11542^{\mathrm{a}}$ & & $\begin{array}{c}\text { Phytocannabinoid } \\
\text { analog }\end{array}$ & Agonist \\
\hline A- $834735^{b}$ & & $\begin{array}{l}\text { Alicyclic } \\
\text { acylindole }\end{array}$ & Agonist \\
\hline AM-2233 & & Benzoylindole & Agonist \\
\hline
\end{tabular}




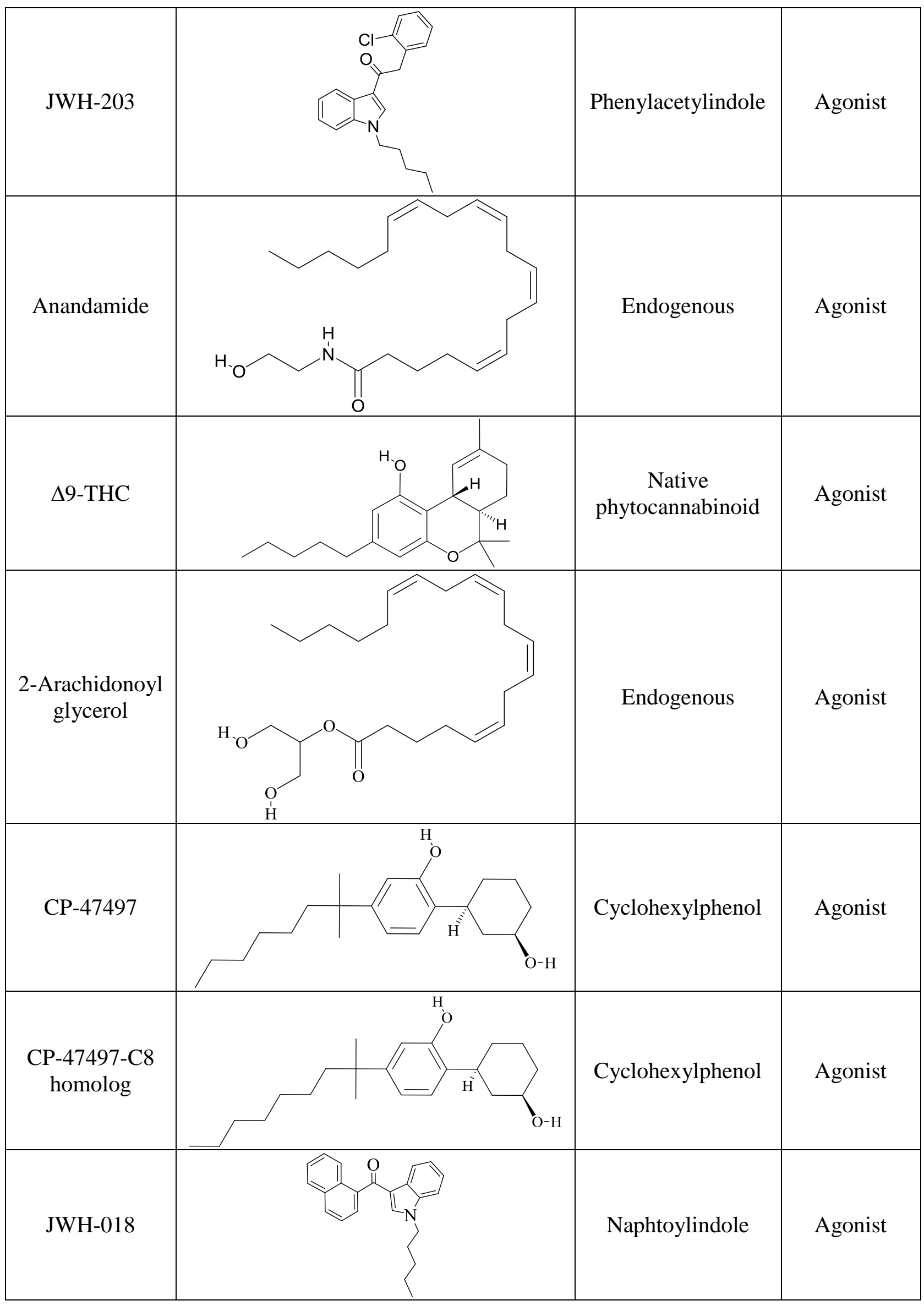




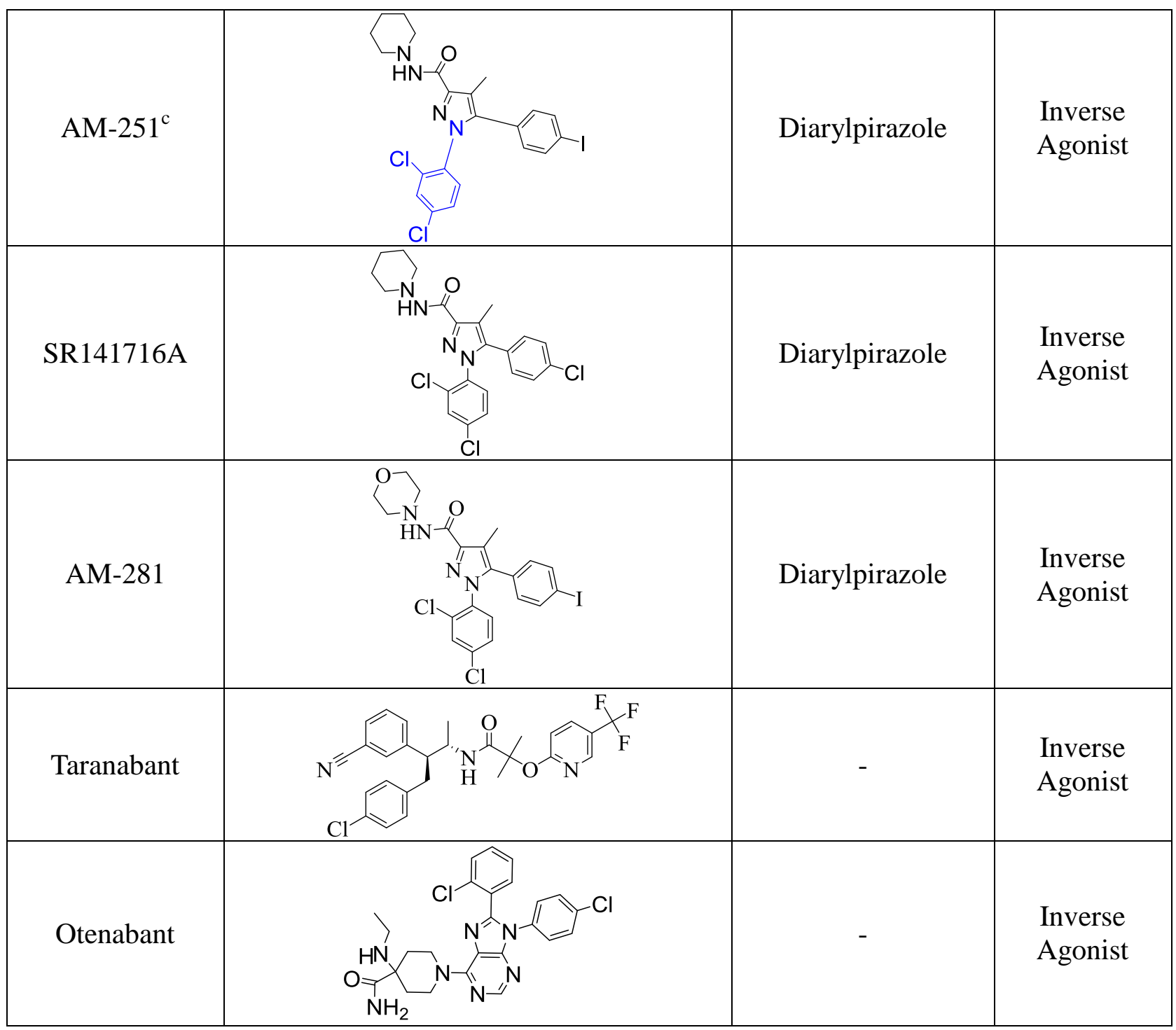

${ }^{a}$ ligand AM11542 from the crystal complex with $\mathrm{CB}_{1}{ }^{6}$

${ }^{\mathrm{b}}$ The ligand's head is shown in green, the linker in orange, the core in magenta and the tail in blue

${ }^{c}$ A subunit of the antagonist ligand that corresponds to agonists' tails at binding is shown in blue

All the ligands from Table 1 were docked into the crystal structure of $\mathrm{CB}_{1}$ in its active conformation (from complex with agonist AM11542, PDB ID: 5XRA). ${ }^{6}$ Initial placement of ligands was done using the Glide $\mathrm{XP}^{13}$ in the region of the agonist AM11542 binding site. Then the modeled placement of ligand was equilibrated via a $50 \mathrm{~ns}$ run of MD simulations of the complex with $\mathrm{CB}_{1}$ at restrains imposed on the atoms of the protein backbone (the Desmond software). ${ }^{16}$ Final locations of agonist and antagonist ligands are shown in Figures $\mathbf{1}$ and 2 , respectively.

In the following text we simplify the description of $\mathrm{CB}_{1}$ agonist structures and divide them into the head and tail only. A generalized new "head" includes the commonly adopted "head", "linker", and "core" (see colored structure A-834735 in Table 1). As seen from Figure 1, all 
agonists are docked in the binding site of $\mathrm{CB}_{1}$ in the same manner. Their heads interacts with hydrophobic residues Phe170, Phe174, Phe177, and His178 of $\alpha$-helix TM2. This interaction, as an important contribution to the agonist binding, was well established earlier. ${ }^{8}$ For brevity we will further name the aromatic interactions of TM2 with the ligand's head as the "hydrophobic lock". As described in ref. 8, in the active conformation of $\mathrm{CB}_{1}$ the TM2 helix moves towards the ligand binding pocket, closing the lock. All ligands have their heads' carbonyl oxygen atoms or hydroxyl groups in the proximity of the side chain of Ser383 to form a non-covalent bond with its hydroxyl group. Another feature of agonists' placements shown in Figure $\mathbf{1}$ is the similar location of their tails. All of them occupy the deep and narrow hydrophobic pocket where the aliphatic chain of agonist AM11542 is located in the crystal structure of complex. ${ }^{6}$ It confirms a noticed important role of this binding pocket. ${ }^{8}$

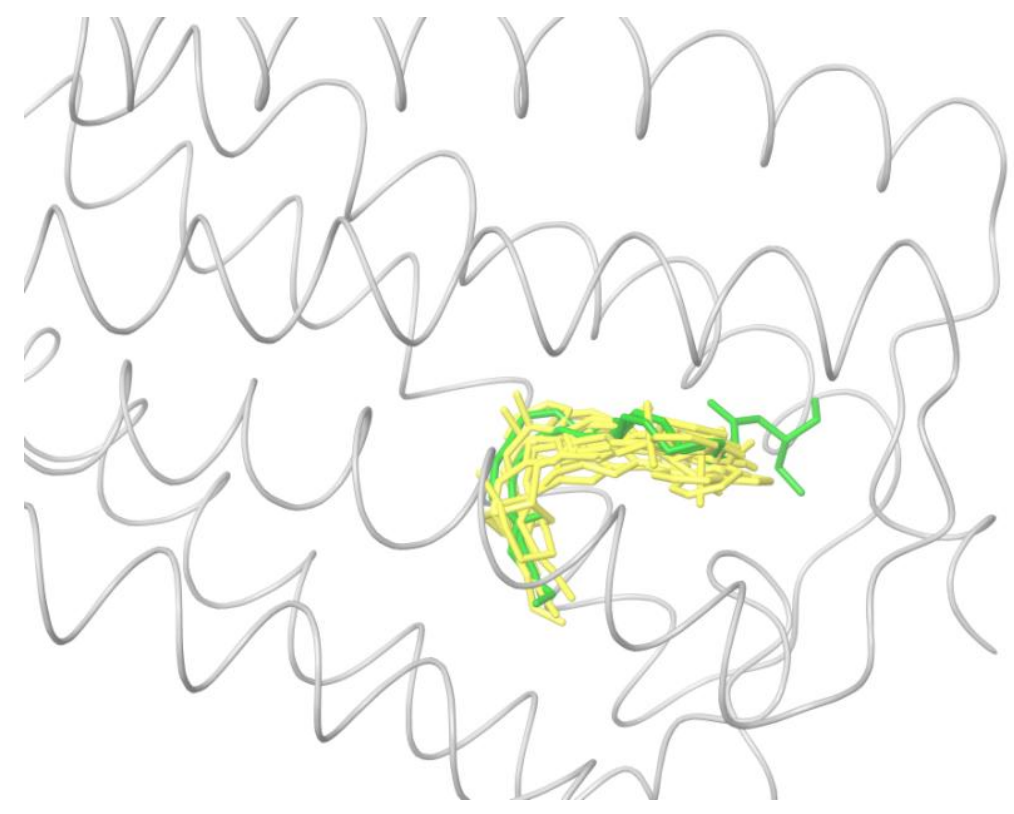

Figure 1. Docking of exogenous agonists from Table 1 into the structure of activated $\mathrm{CB}_{1}$ obtained from the crystal complex with AM11542 (yellow). Docked endogenous cannabinoid (anandomide) is shown in green.

As seen from Figure 2, attempts to dock antagonists into the active conformation of $\mathrm{CB}_{1}$ lead to a different picture. One feature clearly distinguishes the placement of anagonists from the docking of agonists: the tail binding pocket is usually empty in the case of antagonist ligands. Since antagonists possess structural subunits similar to tails of agonists (Table 1), the emptiness of this deep hydrophobic pocket means a high binding energy cost and such docking, even restrained by fixation of the protein backbone chain, cannot be regarded as favorable. 


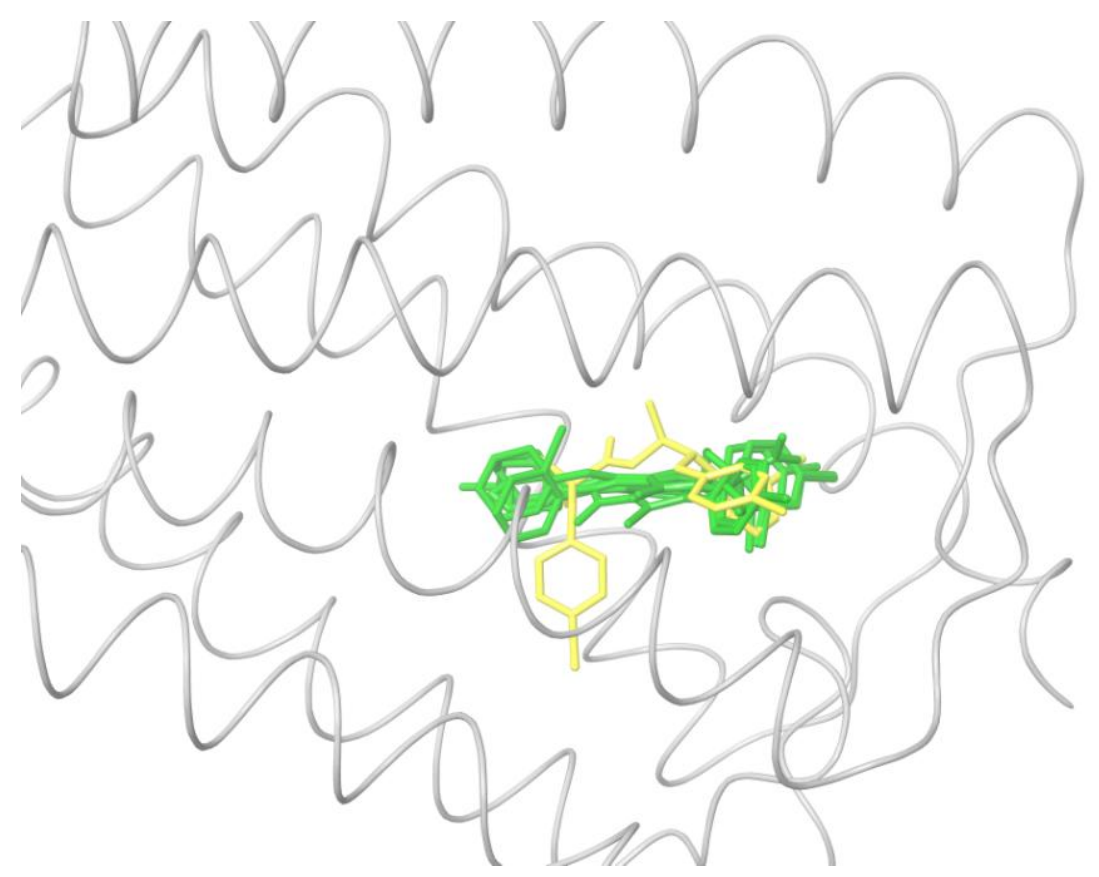

Figure 2. Docking antagonist ligands listed in Table 1 into the structure of activated $\mathrm{CB}_{1}$ obtained from the crystal complex with AM11542. Only one ligand could penetrate into the agonists' tail binding pocket (marked in yellow).

The last conclusion was confirmed when we placed antagonist ligands into inactive $\mathrm{CB}_{1}$ taken from a crystal complex of such an antagonist with the receptor (PDB ID: 5TGZ). ${ }^{7}$ The docking procedure and MD equilibration were similar to ones described above. Results of the antagonists docking into $\mathrm{CB}_{1}$ in an inactive state are shown in Figure 3. One can see that the tail-binding hydrophobic pocket is always occupied by branched subunits of the ligand molecules. Moreover, the placements of all antagonists are quite similar.

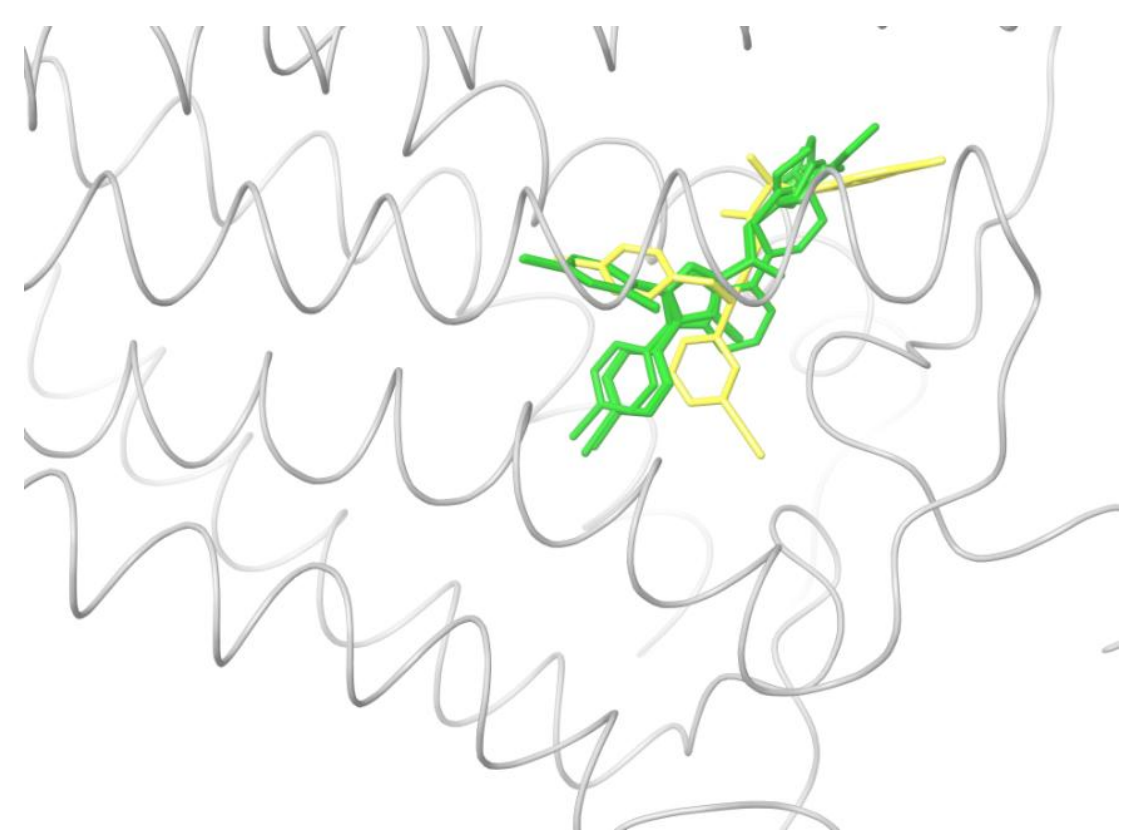

Figure 3. Docking antagonist ligands from Table 1 into the structure of inactivated $\mathrm{CB}_{1}$ taken from crystal complex with antagonist. Similar locations of all ligands except for one marked in yellow. 
A peculiar feature of occupation of the narrow and deep "tail-binding hydrophobic pocket" observed for both agonist and antagonist ligands gives us a possibility to compare the active and inactive conformations of $\mathrm{CB}_{1}$. Since this is the only common feature of agonist and antagonist complexes with $\mathrm{CB}_{1}$, one may assume that protein chains surrounding this peculiar binding hydrophobic pocket should be found in similar conformations. Indeed, they interact with and bind similar hydrophobic subunits of different ligands. The tail-binding hydrophobic pocket is surrounded by extracellular parts of $\alpha$-helices TM3, TM4, and TM5. Thus, on comparing conformations of $\mathrm{CB}_{1}$ from crystal complexes with agonist and antagonist, it is reasonable to superimpose the protein main chains in the two conformations only for those three $\alpha$-helices within the hydrophobic pocket region.

\section{Superimposing active- and inactive-state structures of $C B_{1}$}

In this section we consider such a superposition of the known crystal structures of cannabinoid receptor I that reveals the spatial difference between active and inactive receptor conformations to a maximum extent. As mentioned before, the expected region where the protein surroundings have to be the least different at binding of both agonists and antagonists is the envelope of the deep and narrow hydrophobic pocket accommodating the ligand's tail (Figures 1 and 3 and blue-marked parts of the chemical structures in Table 1). Considering only protein residues within the transmembrane region ${ }^{46,7,15}$ and excluding movable TM6 helix, one gets an approximate picture of the tail-surrounding residues as shown in Figure 4. Certainly, such a choice is arbitrary to some extent and can be varied plus-minus a couple of residues for each chosen fragment of the protein backbone. It is important that the superimposed chains include fragments of only three $\alpha$-helices: TM3, TM4, and TM5. We take residue 195-199 (TM3), 243249 (TM4), and 275-289 (TM5). The last fragment might seem to be chosen too long, as the end of TM5 is shifted at the protein activation. ${ }^{9}$ Anyway, superposition for shorter fragments of TM5 leads to quite similar results. As was mentioned in previous studies ${ }^{8}$ and is further discussed in the present work, the very spatial packing of TMs is rigid enough for each conformation of $\mathrm{CB}_{1}$ determined by the ligand bound: all seven transmembrane $\alpha$-helices are glued by numerous mutual molecular contacts to form the central channel. Taking this into account, we can explain the inclusion of a short fragment of TM4 rather for increasing the structurally stable region chosen, as this fragment adjoins to the region of residues of TM3 and TM5 interacting with the 
ligand's tail. Besides, the near loop TM4-TM5 is very stiff: it involves an S-S bridge. Further we justify the choice of this structural stability region, considering its peculiar structural features.

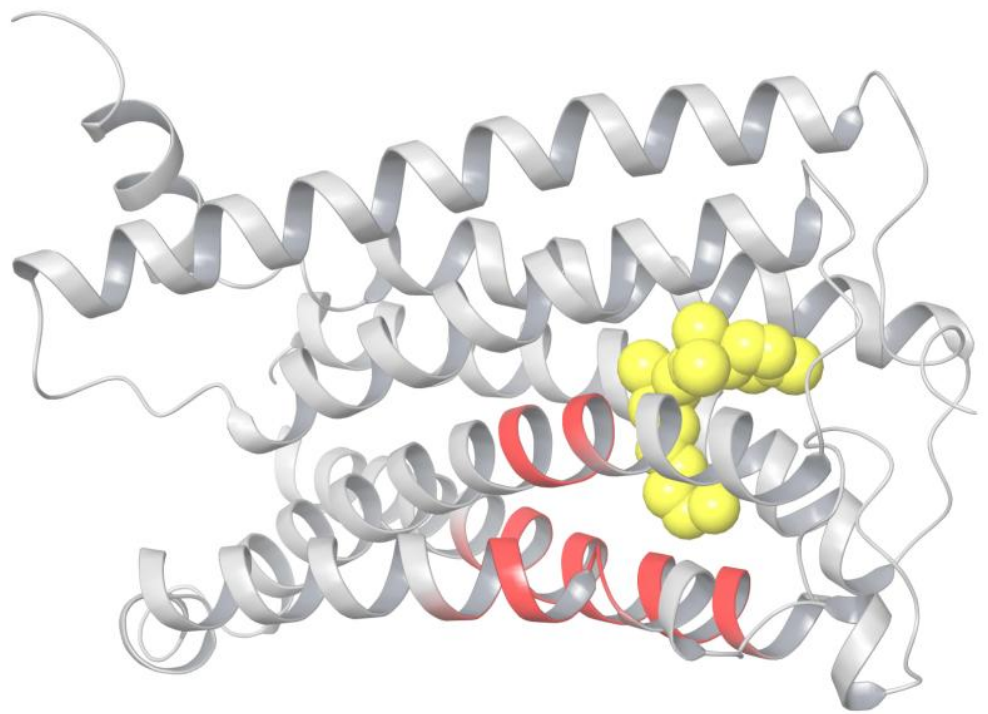

Figure 4. Intramembrane residues of TM3, TM4 and TM5 (red) near the tail binding pocket in the active state conformation of $\mathrm{CB}_{1}$. The agonist ligand is shown in yellow.

A picture of superposition of the crystal structure of $\mathrm{CB}_{1}$ in its active-state conformation (from PDB entry 5XRA) on its inactive state (from PDB entry 5TGZ) is shown in Figure 5. Superimposed regions to minimize the RMSD of the main chains are marked in yellow, observed discrepancies in packing $\alpha$-helices TM1, TM2, TM6, TM7, and at the end of TM5 are depicted by arrows. Among all seven $\alpha$-helices, residues of TM1 and TM4 have no direct interactions with agonist ligand.

The structural changes during activation of $\mathrm{CB}_{1}$ are rather evident in Figure 5. Indeed, the "downward" movement of TM1 is due to removal of the "upper long arm" of the antagonist ligand (see Figure 3). The "downward" movement of TM2 is caused by the attraction of residues F170, F174, F177, and H178 of the TM2's "hydrophobic lock" to the "generalized" head of the agonist ligand. This interaction was well described earlier. ${ }^{8}$ The movement of TM6 provides the allosteric modulation of the G-protein binding. The immobile TM3, TM4, and most part of TM5 we will further call the "basement". A key movement causing the allosteric shift of TM6 is a conformational change of neighboring TM7. In fact, TM6 is just pressed out by the movement of TM7 towards the basement (TM5). At least in part, this movement of TM7 is caused by similar movements of adjoining TM2 and TM1. TM7 and TM2 have numerous molecular contacts of side chains of their residues (as well as other neighboring $\alpha$-helices). All 
helices form the cylindrical shell of the central transmembrane channel of the receptor and this shell is rigid enough due to self-consistency of the packing of adjoining $\alpha$-helices.

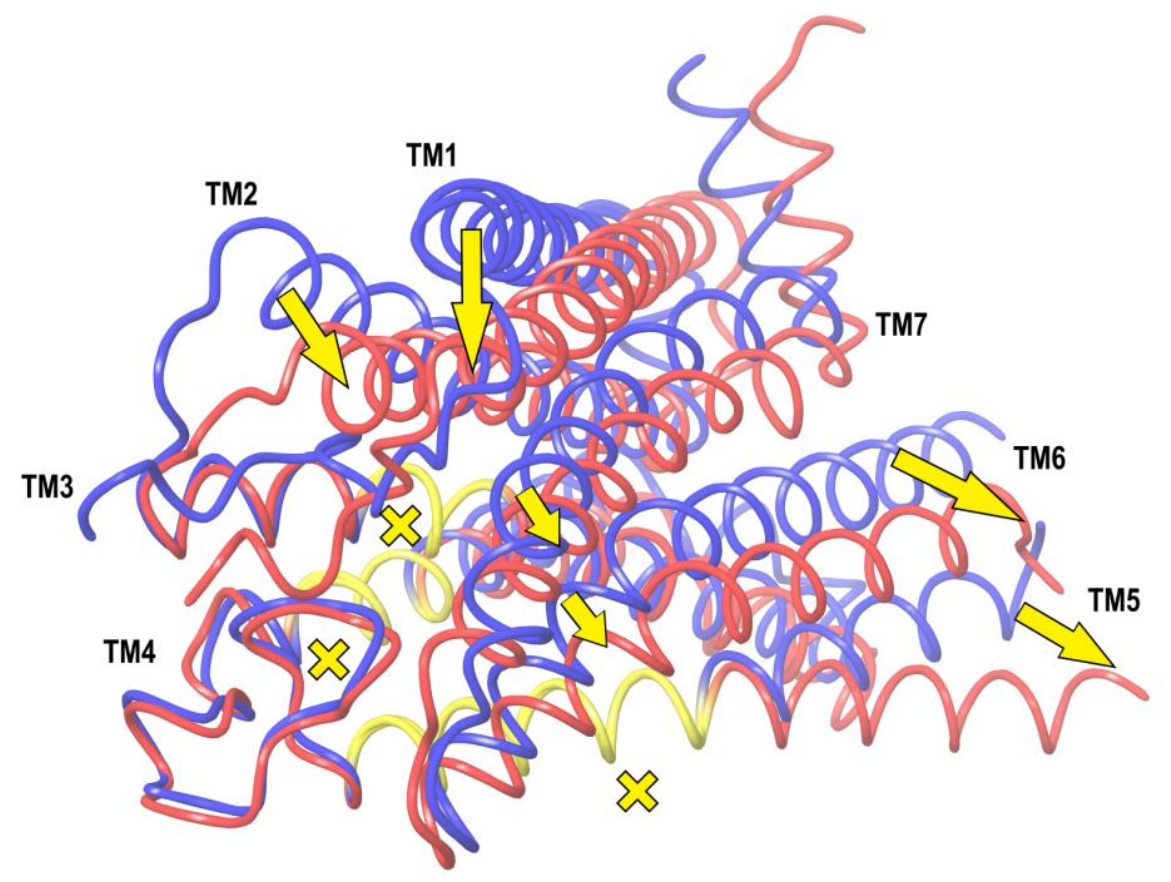

Figure 5. A superposition of crystal structures of $\mathrm{CB}_{1}$ in its inactive (blue) and active (red) states. The RMSD of the backbone atoms are minimized in the region marked in yellow. Arrows indicate movements of the transmembrane $\alpha$-helices, crosses denote coincided ones.

How self-consistent the two conformations of $\mathrm{CB}_{1}$ are, can be verified by $\mathrm{MD}$ simulations. We have carried out a simple modeling experiment to check how densely each conformation is packed in terms of interactions between neighboring $\alpha$-helices. Replacement of TM6 (residues 332-364) taken in its active-state conformation by TM6 in the inactive-state conformation (in respect to TM5 and TM7) and vice versa led to restored original conformation for less than $50 \mathrm{~ns}$ MD simulations of $\mathrm{CB}_{1}$ immersed into the membrane. Such computer simulations for replaced TM6 were repeated for models with ligands bound and when ligands were removed from the structure of the complex. The stability of the original conformation did not depend on the presence of the ligand.

Looking at Figure 5, one can raise a simple important question. If one removes the antagonist ligand from the receptor's channel, some movement of TM1 in the direction shown in Figure 5 should obviously take place. As the ligand surroundings in the receptor channel are hydrophobic, the vast vacant space cannot just be filled with the solvent. Then some movement of TM2 similar to one shown in Figure 5 and, perhaps, of TM7 should also occur. But it cannot result in the picture corresponding to the active state of $\mathrm{CB}_{1}$, otherwise an empty channel would lead to activated receptor. Thus, the question of which interactions of agonist ligand with the 
receptor finalize the protein transformation into its active state remains unresolved. In the following we describe a study dedicated to this quest.

\section{Construction of a model of a sensitive-to-agonist-binding conformation of "unliganded" $C B_{1}$}

The structures of the transmembrane domain of $\mathrm{CB}_{1}$ in its active-state and inactive-state conformations (shown in Figure 5) are coherent, self-consistent and metastable. It can easily be demonstrated via MD simulations. If one removes the antagonist ligand from the inactive structure (PDB ID: 5TGZ) and starts MD simulations of the transmembrane domain of the remaining empty receptor (immersed into a membrane), during many hundreds of nanoseconds of a MD run, the protein tertiary structure remains mainly intact except for denser packing of TM1 due to the ligand removal. If one docks agonist ligand into this structure, no transformation into the active-state conformation known from crystallographic data will occur for any reasonable time of the simulation run (more than a thousand of nanoseconds). Equally, if one just removes agonist ligand from the active-state structure of $\mathrm{CB}_{1}$, even a very long run of $\mathrm{MD}$ simulations of such empty receptor in membrane indicates no visible structure transformation leading to disappearance of the active-state conformation of TM6. This means that high potential barriers have to be overcome at forming the conformation of $\mathrm{CB}_{1}$ caused by protein-ligand interactions. Such barriers should be distinguished from the free energy barrier at transfer of a ligand molecule from bulk solution into the receptor's orthosteric binding pocket - it is also high and such a transfer can only be explored by a method of metadynamics simulations. ${ }^{9}$ It is clear that the barrier of receptor's structural transformation is caused by the numerous mutual interactions of adjoining residues of neighboring $\alpha$-helices of the densely packed channel protein.

As can be seen in Figure 5, the primary cause of the structural transformation of $\mathrm{CB}_{1}$ from its inactive to active states is the TM2 movement. To some extent, we can also add interactions between the agonist ligand and TM6 or TM7. Displacements of TM1 and the intracellular parts of TM5 and TM6 are far beyond the direct interactions with the agonist ligand, therefore we regard them as secondary ones. But those very outward movements of TM6 and TM5 are of importance since they provide the allosteric modulation at G-protein binding. One can assume that for building a model of apo receptor sensitive enough to be easily transformed into the final active conformation by agonist bound, it is necessary to diminish the high potential barrier at the shift of TM2 shown in Figure 5. This shift is caused by the hydrophobic lock interactions with the head of ligand - one of the main sites of ligand binding. ${ }^{8}$ The barrier itself is caused by dense 
TM2-TM3 and TM2-TM1 interactions along the $\alpha$-helices at the corresponding movement of TM1.

The desired model of the seven-transmembrane (7TM) helices domain of $\mathrm{CB}_{1}$ should include the following features. TM6 and TM7 still remain in inactive-state conformation, but geometry of TM1 and TM2 is noticeably closer to their final active conformation at binding of agonist ligand. Such a model can be built in several ways. For example, after removing antagonist ligand from the inactive-state crystal complex, one can apply a metadynamics simulation procedure to force the shift of TM2 and TM1 towards their desired locations in active conformation of $\mathrm{CB}_{1}$. Then the void space of the hydrophobic channel in its extracellular region will be noticeably diminished. This metadynamics simulation procedure is described in the Methods section and results are shown in Figure S1 (Supporting Information). In our study, a different procedure has also been applied and we initially modeled the structure of "unliganded receptor" in a bit more complicated way. It involves simple molecular modeling and a series of computer MD simulations to equilibrate receptor without ligand in the membrane environment.

At first we built a chimeric model of the 7TM domain of $\mathrm{CB}_{1}$, where the conformation of residues before Ala244 was taken from the active conformation (PDB ID: 5XRA) and residues from Ala244 were constructed according to receptor's inactive state (PDB ID: 5TGZ). It should be noted that between residues 243 and 249 both conformations can be superimposed at low RMSD of the backbone atoms (see above). In other words, TM1, TM2, TM3, and a part of TM4 were taken from the active state, whereas TM5, TM6, and TM7 from the inactive one. Such a structure can be easily built without serious interceptions of residue atoms if the backbone fitting involves the abovementioned fragments of TM3, TM4, and TM5 (marked in yellow in Figure 5).

The second step was equilibration of this chimeric model of apo receptor. It included a series of MD simulations of this model. Initial two simulation runs of $200 \mathrm{~ns}$ each were done at geometry restraints applied. At the first run, the "active-conformation" part of the backbone atoms was subjected to the restraining potential preventing their significant shift from initial positions. These simulations showed that the second part of the structure (after Ala244) had kept their inactive conformation. At the second $200 \mathrm{~ns}$ MD run, the "inactive-conformation" part was restrained in movement of the protein backbone atoms, whereas the protein chain preceding Ala244 was completely released. Its initial active conformation notably changed after the simulation. At the third $200 \mathrm{~ns} \mathrm{MD}$ run, the entire 7TM domain of $\mathrm{CB}_{1}$ was released. Anyway, after that $\alpha$-helices TM5, TM6 and TM7 retained their inactive conformations such as observed in the crystal structure with antagonist ligand bound. The very shape of the protein has changed a little, except for $\alpha$-helix TM1 appeared to be bent at one place. The final part of modeling 
included a slight correction of TM1 placement to restore its integrity. At the end the entire model was equilibrated again by means of a 200 ns MD run. And finally, the (meta)stability of this "empty-receptor model" was verified via a long-time MD run of about 500 ns length (all simulations in the membrane environment).

A superposition of the active-state, inactive-state, and modeled apo conformations of $\mathrm{CB}_{1}$ are shown in Figure 6. As seen, $\alpha$-helices TM3, TM4, TM5, TM6, and TM7 in the "unligandedmodel" conformation almost coincide with those in the inactive-state conformation. On the contrary, TM2 is quite close to (but not coincides with) TM2 in the active-state conformation. Helix TM1 is found somewhere between its conformations in the active and inactive states.

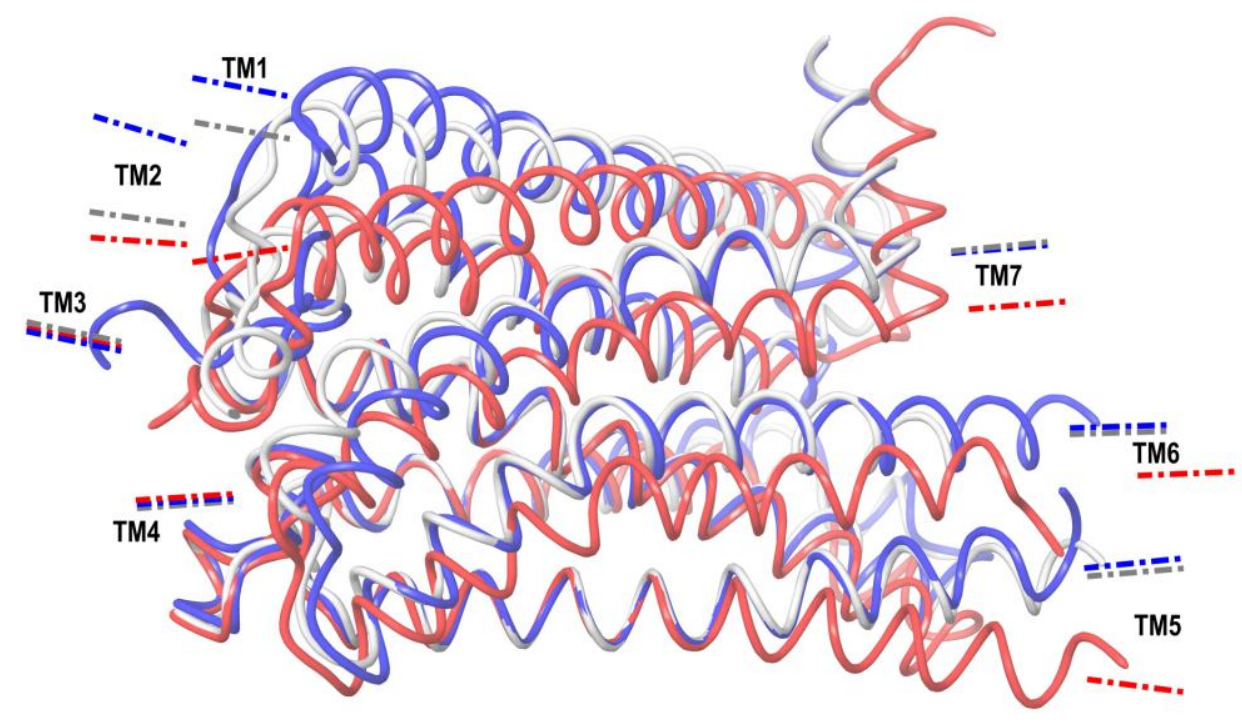

Figure 6. Comparison of $\mathrm{CB}_{1}$ structures from the crystal structure of complex with antagonist (blue, inactive-state conformation), from the crystal structure of complex with agonist (red, active-state conformation), and modeled "unliganded-receptor" structure (white). Directions of axes of the $\alpha$-helices are depicted as short dashed-dotted lines.

\section{Simulated transformation of the inactive "empty receptor" into the active state of $\mathrm{CB}_{1}$ at agonist ligand binding}

The molecule of $\mathrm{CB}_{1}$ agonist AM11542 from the reported X-ray structure of complex ${ }^{6}$ was docked into the "model of unliganded $\mathrm{CB}_{1}$ " described in the previous section. (The coordinate file of the resulting complex is given in the Supporting Information.) As seen from structure comparison shown in Figure 6, such a complex appears to be structurally not too much different from the crystal complex from X-ray data and the ligand placement does not undergo serious steric collisions. Results of $400 \mathrm{~ns}$ MD simulations of the new complex of $\mathrm{CB}_{1}$ in the "unliganded-receptor" conformation with AM11542 are shown in Figure 7. 
One can see that all the receptor structure transformations into the active state (Figure 5) have occurred after this MD run. (Actually, the crucial ones such as movement of TM7 pressing out the allosteric $\alpha$-helix TM6 take about $200 \mathrm{~ns}$ of simulation time.) We should not be confused by the incomplete outward movement of TM6 at its breakage point in the intracellular area, as a part of the chain (307-338) is removed from the model. As a result of this model distortion, a rather hydrophobic intracellular entrance into the channel appears to be exposed to solvent and a similar bent of TM6 near the breakage of the chain (residues preceding Leu345) can be observed for prolonged MD simulations of the crystal complex of $\mathrm{CB}_{1}$ with $\mathrm{AM} 11542$. Another thing is of crucial importance: exact coincidence of the final location of TM7 with its geometry in the crystal complex and long coincidence of TM6 (residues 345 - 367) with TM6 from the activestate X-ray structure.

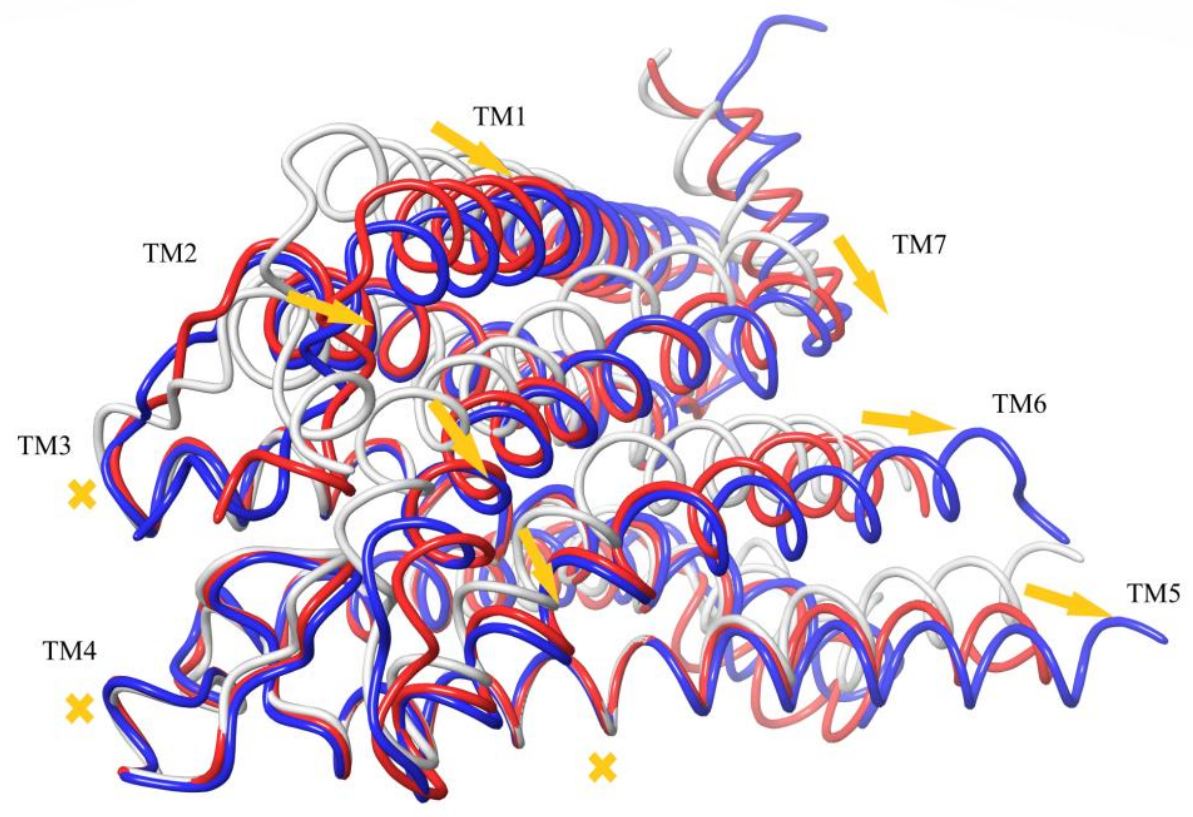

Figure 7. Results of $\mathrm{MD}$ simulations of the $\mathrm{CB}_{1}$ in modeled "unliganded-receptor" conformation with docked agonist AM11542. The "unliganded-receptor model" is shown in grey, the final conformation after a $400 \mathrm{~ns}$ MD run in red. The structure of $\mathrm{CB}_{1}$ in the active state from crystal complex with AM11542 ${ }^{6}$ is shown in blue. The structures are superimposed at minimization of RMSD of protein backbone atoms of the "basement" (residues 195199, 243-249, and 275-289). The $\alpha$-helix movements are depicted by arrows. Immovable $\alpha$-helices of the "basement" are marked by crosses.

Among other important structural changes we should mention the move of TM1 towards its placement in the crystal structure and a displacement of TM2 due to accommodation of its "hydrophobic lock" interacting with the head of the agonist ligand. In Figure 7 all movements of 
$\alpha$-helices are shown in respect to the immovable "basement" of TM3, TM4, and TM5 marked in Figures 4 and 5. Residues of this "basement" interact with the tail of ligand. ${ }^{47}$

One feature of the initial ligand docking for this complex was useful for further research: there was only a tiny movement of the ligand molecule in respect to the "basement" after a 400 ns MD run (less than $1 \AA$ ). The backbone chain of the "basement" itself was immobile as well (RMSD less than $0.5 \AA$ ). The resulting structure is not changed if heavy atoms of the backbone chain of the "basement" are spatially anchored by a restraining potential. The MD simulations were repeated when heavy atoms of both the ligand and backbone of the basement were fixed by the restraining potential. It led to a receptor conformation that was close to the structure of the ligand-receptor complex obtained from the simulations with free ligand. It is not so if the ligand is structurally modified (see below).

The obtained transformation of the $7 \mathrm{TM}$ domain of $\mathrm{CB}_{1}$ into the conformation of its active state allows us to explore the entire mechanism of the enzymatic reaction with agonist leading to the allosteric modulation. It can be studied in a series of particular MD simulations with this model.

\section{The $C B_{1}$ activation molecular mechanism}

The activation of $\mathrm{CB}_{1}$ at the agonist ligand binding described in the previous section occurs within a short simulation time $(\sim 200 \mathrm{~ns})$ due to the fact that in the inactive-state metastable apo conformation the potential barrier of closing the hydrophobic lock is essentially diminished in comparison with, e.g., the conformation of $\mathrm{CB}_{1}$ taken from the crystal complex with antagonist ligand. It is important to note that this entire transformation is reversible. Indeed, when we removed the agonist ligand from the final active structure shown in Figure 7 (in red color) and started MD simulations of the empty receptor, the original "unliganded-receptor model" was restored in a $200 \mathrm{~ns}$ MD run. This directly proves that in these simulations the activation is entirely driven by the protein-ligand interactions.

It should be noted that we could not obtain the inactive-state conformation of the unliganded receptor directly by $\mathrm{MD}$ simulations of its active conformation from the crystal complex ${ }^{6}$ when agonist ligand is removed. Simulation experiments showed that additional potential barriers were in the intracellular region of model distortion, where the protein chain is broken for $\alpha$-helices TM5, TM6, and TM7. This part of the X-ray data structure of the active-state receptor was replaced by the active-state structure obtained in the simulations of our "unliganded-receptor model" with agonist ligand (residues 195-414). After that the inactive "unliganded-receptor model" reappeared in $200 \mathrm{~ns}$ MD simulations of the empty receptor in membrane despite some 
structural difference in TM1 and slight difference in the backbone chain of TM2 (red and blue structures in Figure 7).

The second computational experiment confirmed the crucial role of the "hydrophobic lock". We started the MD simulation run for the "unliganded-receptor" conformation after docking AM11542 into its binding pocket, but at that time the protein backbone atoms of the TM3-TM5 "basement" (residues 195-199, 243-249, and 275-289) and, on the other hand, of the entire $\alpha$ helix TM2 were restrained to move due to an imposed restraining potential. (Further in the text we call such a restriction as "fixation" of a protein backbone chain fragment or other atomic group.) Thus, we "fixed" TM2 in respect to the "basement", preventing the shift of TM2 shown in Figure 7. As a result of a long MD simulation run, no active conformation was observed. In fact, the structure of the complex remained intact. Therefore, the shift of TM2 is of crucial importance for the receptor activation. The TM2 displacement (from $1.5 \AA$ for Ala160 to $3.8 \AA$ for Val171) is smaller than the movement of adjoining TM7 (3.5-5.0 $\AA$ for residues 377-398) and TM1 (up to $7.7 \AA$ for Ser114). This shift is the final accommodation of the "hydrophobic lock" (F170, F174, F177, H178) in respect to the "basement". Thus, an obstacle for this final accommodation totally blocks the receptor's structural rearrangement.

The role of the "hydrophobic lock" accommodation in respect to the geometry of the "basement" can easily be proven by several other simulation experiments. In the first one we remove the entire tail of the ligand and only its "head" remains. But now the "head" is spatially fixed at the MD run, as well as the receptor's "basement". (As mentioned above, after simulations with complete AM11542, the ligand is almost immovable relative to the "basement".) Results of 200 ns MD simulations of the "unliganded-receptor model" with ligand's head at such restrains are close to those shown in Figure 7: the active-state conformation appears. On the contrary, if we release the head of AM11542 and the ligand has no "tail", no active conformation of $\mathrm{CB}_{1}$ is obtained. Instead, the "head" interacts with the "hydrophobic lock" of TM2 and notably moves from its initial placement. And finally, if we fix the "tail" of the ligand in respect to the fixed "basement" and remove the ligand's "head", there is no active conformation of the receptor after MD simulations either.

Thus, the entire molecular mechanism of $\mathrm{CB}_{1}$ interaction with its endogenous ligands looks very simple. The ligand "head" attraction to the "hydrophobic lock" of TM2 together with ligand's tail binding to the "basement" ( $\alpha$-helices TM3, TM4, and TM5) force $\alpha$-helices TM1 and TM7 to move towards the "basement" mainly due to a shift of TM2 in respect to this "basement". This contraction of the "cylindrical channel shell" of TMs pushes out $\alpha$-helix TM6, providing the allosteric modulation of G-protein binding. This scheme is shown in Figure 8. The shift of the backbone of TM2 is towards TM7 and almost tangential to the "basement". The 
movement of the side chains of TM2's "hydrophobic lock" (especially Phe170 and Phe174 in the case of ligand AM11542) pushes neighboring residues of TM7 towards the "basement" along the direction of the ligand's tail aliphatic chain. Direct interactions of TM7 residues with the ligand head (such as a hydrogen bond of Ser383) also take a part in this shift of TM7 towards the basement.

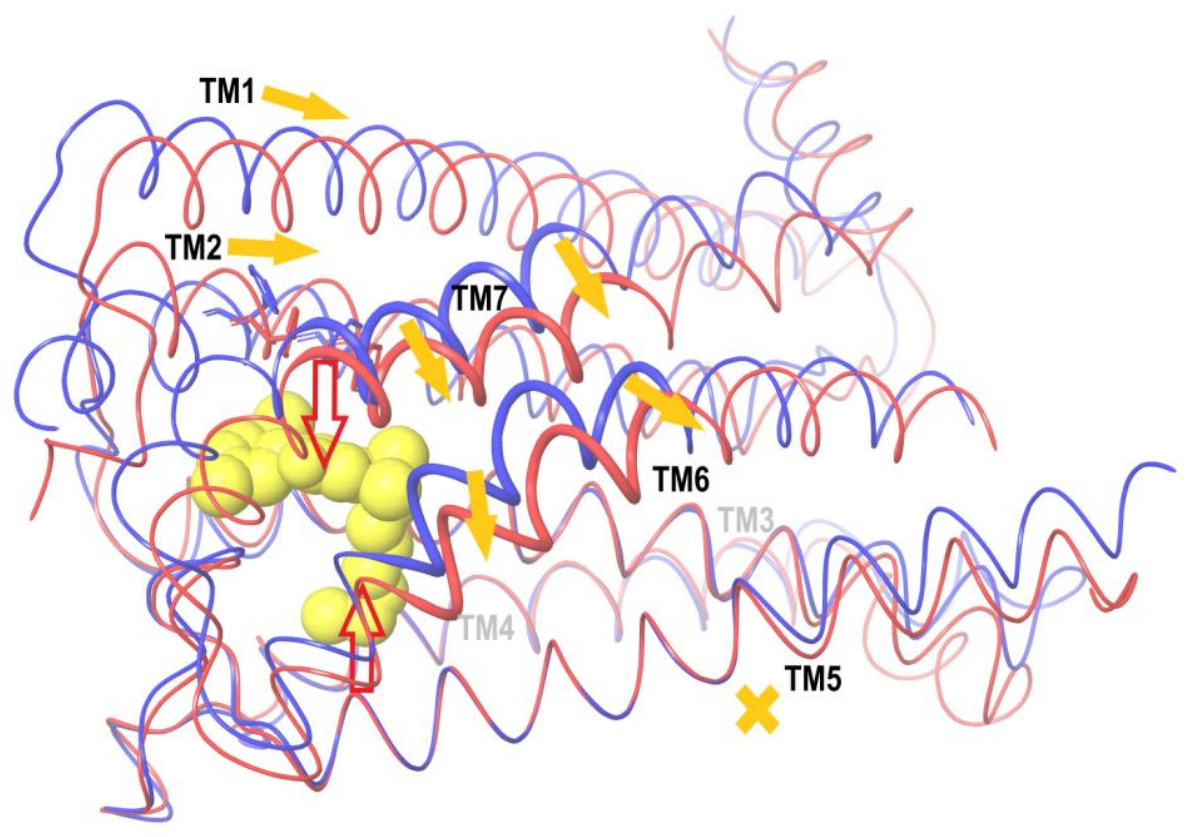

Figure 8. An explanation of the activation mechanism. The AM11542 agonist ligand molecule is shown in yellow. Red arrows denote hydrophobic interactions of the ligand "tail" with TM3 and TM5 and of the ligand "head" with the "hydrophobic lock" of TM2 (its residues F170 and F174 are shown). The backbone for the "unliganded-receptor model" conformation is marked in blue, results of MD simulations of this model with the ligand is shown in red. The depicted thick part of the backbone of TM6 and TM7 marks residues chosen for RMSD evaluation to detect the receptor activation. See the text for further details.

At this point we should carefully examine the previously determined "basement" which interacts with the alkyl chain of the ligand tail. Indeed, the three $\alpha$-helices TM3, TM4, and TM5 form a very stiff and densely packed structure. The hydrophobic core of their mutual interactions includes the following residues: 197-212 (TM3) contacting with 275-289 (TM5); 190-201 (TM3) contacting with 244-251 (TM4); 247-251 (TM4) contacting with 275-279 (TM5). In fact, TM3TM4 hydrophobic interactions involve the entire length of each $\alpha$-helix. Besides, TM4 is connected to TM5 by a hydrogen bond Tyr275-Ile247. The extracellular loop TM4-TM5 is fixed by S-S bridge Cys257 - Cys264. The dense packing of TM3 and TM4 is maintained by additional interaction Asp213-Tyr224 at the intracellular loop connecting them. In the suggested activation mechanism there is a preferential direction along the alkyl chain of the tail of ligand 
AM11542 perpendicular to the approximate plane of its head's cycles. Along this line of sight, projections of axes of $\alpha$-helices TM4, TM3, and TM2 in the active state conformation cross at approximately one point, giving the "loaded nodes": residues 244-251 (TM4) against 194-200 (TM3) against 166-173 (TM2) (Figure 9A). We assume "loaded", as a tension is expected (red arrows in Figure 8) approximately along this line. An indirect evidence of this loaded node of TM3 (which is between TM2 and TM4 in this projection) is the fact that backbone chain of $\alpha$ helix TM3 is reinforced exactly in this region by three (!) additional hydrogen bonds: Thr197 Leu193; Ser199-Gly195, and Thr201-Thr197. Besides, TM4 conformation is strengthened by Pro251. Looking at the residues of TM5 neighboring to the two "loaded nodes" of TM3 (194200) and TM4 (244-251), it is seen that they involve residues 275-283. And we again find there reinforcing of the backbone by an additional hydrogen bond: Ile280-Ser284. Thus, according to the crystal structure of $\mathrm{CB}_{1}$ in its active-state conformation, we see a "reinforced" basement of heavily packed and interacting helices, where conformation of residues 194-200, 244-251, and 275-283 is expected to be very stable. Comparing to our initial choice for structures superposition (195-199, 243-249, and 275-289), one can see that they coincide except for longer length of the stable part of TM5.

It should be noted that the kink point of TM5 in $\mathrm{CB}_{1}$ is Leu286. For other class A GPCR proteins there is a proline residue at this position and it is a kink point of TM5. ${ }^{8}$ It is important to note that this residue is at crossing of TM5 and TM6 axes projections in inactive state along the line of tension - in active state TM6 is pressed out and moves outwards. It not only justifies our initial choice of the superposition residue intervals, but also casts some light how to detect the active state in the result of simulations. As mentioned above, the very beginning of TM6 is subjected to interactions due to the breakage of the protein backbone in the intracellular domain of the simulated model and we even truncated TM6 to Ile339 to avoid a computationally consuming breakage of the "ionic lock" Arg214). But the active state of $\mathrm{CB}_{1}$ should be indicated by the movement of TM6 and TM7 in respect to TM5 rather in the extracellular and membrane regions than at the very beginning of TM6 where the simulation model is not correct due to the breakage of the protein main chain. Thus, looking at Figure 7, it is natural to calculate the RMSD of the TM6 and TM7 displacement from the inactive state of our "unliganded-receptor model" to the active state, counting residues of TM6 and TM7 lying above TM5 before its kink point Leu286. "Above" means the direction of the tension approximately along the alkyl chain of the ligand tail (Figure 9B). It gives an approximate interval of residues Val351 - Thr391. The "helical part" of this interval is marked in Figure 8 and it was used for RMSD calculations for comparison with the active- and inactive-state crystal structures of the receptor. Results of MD simulations for 
different ligand modifications or mutations are collected in Table 2, where the active state is detected via RMSD in this interval of residues when alignment of $\mathrm{C} \alpha$ atoms of compared structures includes the "basement" residues 195-199, 243-249, and 275-289.

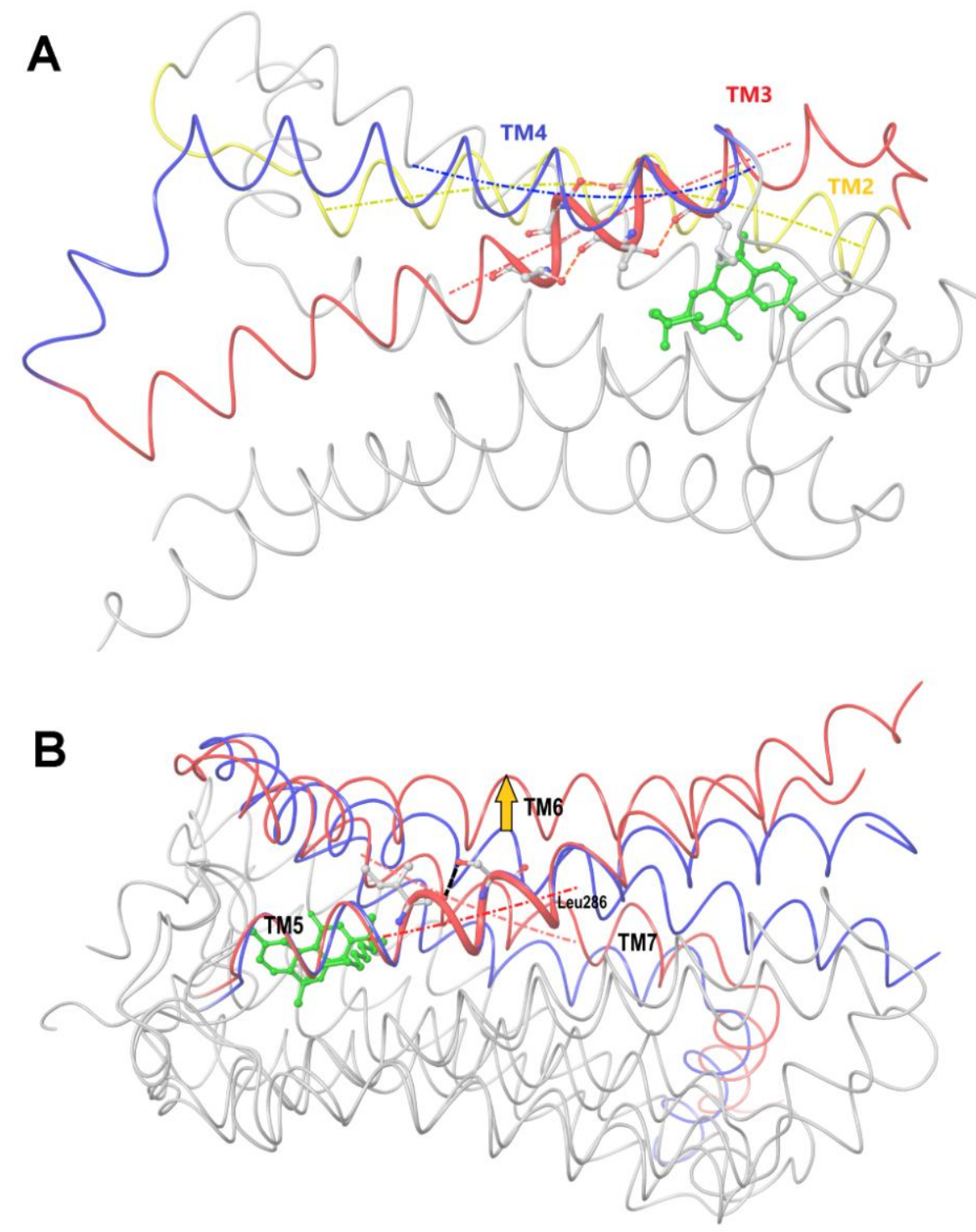

Figure 9. (A) Approximate projection of the mean force acting on the TM3 - TM4 part of the receptor's hydrophobic core (TM3-TM5) due to TM2 - ligand head interactions along the aliphatic chain of the ligand tail (looking towards us) and perpendicular to the planar ligand head. Alpha helix TM3 (red) is sandwiched between TM2 (yellow) and TM4 (blue). Its part reinforced by three additional hydrogen bonds is marked thick. Axes of the $\alpha$-helices are depicted by dashed-dotted curves. (B) Approximate projection of the mean force acting on the TM5 part of the receptor's hydrophobic core due to movement of TM7 towards TM5. The projection is along the aliphatic chain of the ligand tail (looking towards us) and perpendicular to the planar ligand head. Superposition of the inactive-state (blue) on active-state (red) conformations of $\mathrm{CB}_{1}$ taken from crystal complexes of $\mathrm{CB}_{1}$ with antagonist and agonist ligands. The structures are superimposed at the "basement" residues 195-199 (TM3), 243-249 (TM4), and 275-289 (TM5). Axes of helices TM7 (in the active state) and TM5 are shown as dashed-dotted lines. The agonist ligand AM11542 is colored in green. A "reinforcing" hydrogen bond between the side chain of Ser284 and Ile280 is shown as dashed line. The backbone of the loaded part of TM5 is marked thick. Leu286 in CB $_{1}$ 
corresponds to the proline residue in other A-GPCR receptors. This residue separates TM5 into the rigid and flexible parts. Due to ligand-protein interactions, the mean force acts on TM7 towards fixed TM5 along the line of the observer's sight, pressing TM6 out (yellow arrow).

TABLE 2: RMSD between simulated conformations of the receptor and its conformations in active and inactive states from crystal complexes

\begin{tabular}{|c|c|c|}
\hline \multirow[t]{2}{*}{ Simulation description } & \multicolumn{2}{|c|}{$\begin{array}{l}\mathrm{C}_{\alpha} \text { RMSD / max } \\
\text { distance }(\AA) \text { from }^{\text {a }}\end{array}$} \\
\hline & active & inactive \\
\hline 5TGZ.pdb crystal complex with antagonist ${ }^{7}$ & $3.7 / 5.4$ & $0.0 / 0.0$ \\
\hline 5XRA.pdb crystal complex with agonist ${ }^{6}$ & $0.0 / 0.0$ & $3.7 / 5.4$ \\
\hline 5XR8.pdb crystal complex with agonist ${ }^{6}$ & $0.4 / 0.9$ & $3.6 / 5.3$ \\
\hline The model ${ }^{\mathrm{b}}$ & $4.1 / 5.7$ & $0.9 / 1.6$ \\
\hline The model (with no ligand) $(400 \mathrm{~ns})^{\mathrm{c}}$ & $4.1 / 5.4$ & $1.3 / 2.0$ \\
\hline The model with free AM11542 (400 ns) & $1.3 / 2.2$ & $2.9 / 4.3$ \\
\hline The model with fixed ${ }^{\mathrm{d}}$ AM11542 (400 ns) & $1.8 / 2.6$ & $2.4 / 3.3$ \\
\hline The model with fixed AM11542 without its head (200 ns) & $3.1 / 5.2$ & $1.7 / 2.5$ \\
\hline The model with fixed AM11542 without its tail (200 ns) & $2.7 / 3.6$ & $2.2 / 3.1$ \\
\hline The model with precisely fixed AM11542 without its tail $(200 \mathrm{~ns})^{\mathrm{e}}$ & $1.4 / 2.3$ & $3.1 / 3.9$ \\
\hline The model with free AM11542 without its tail (200 ns) & $4.9 / 5.7$ & $1.9 / 3.5$ \\
\hline The model with free AM11542 with truncated C4 tail (200 ns) & $4.4 / 5.9$ & $1.7 / 2.9$ \\
\hline The model with free AM11542 with truncated C5 tail (200 ns) & $0.9 / 1.6$ & $3.2 / 4.5$ \\
\hline $\begin{array}{l}\text { The model, "reverse" simulations of the active complex obtained by } 400 \mathrm{~ns} \\
\text { MD run of the model with AM11542: AM11542 is removed ( } 400 \mathrm{~ns})\end{array}$ & $3.5 / 4.9$ & $2.1 / 3.4$ \\
\hline 5XRA.pdb crystal complex with AM11542 removed (200 ns) & $0.7 / 1.3$ & $3.8 / 5.5$ \\
\hline The model with fixed AM11542, TM2 is fixed (200 ns) & $3.2 / 4.7$ & $1.5 / 2.6$ \\
\hline $\begin{array}{l}\text { The model, "reverse" simulations of the active complex obtained by } 400 \mathrm{~ns} \\
\text { MD run of the model with AM11542: AM11542 is removed along with } \\
\text { Phe } 200 \text { mutated to Gly200 ( } 400 \mathrm{~ns}) \text { f }\end{array}$ & $3.2 / 4.9$ & $1.2 / 1.6$ \\
\hline The model with fixed AM11542, Trp356 is mutated to Gly356 (200 ns) & $2.2 / 3.9$ & $2.0 / 3.0$ \\
\hline The model with free AM11542, Trp356 is mutated to Gly356 (200 ns) & $2.8 / 4.2$ & $1.5 / 2.1$ \\
\hline The model with free AM11542, Phe200 is mutated to Gly200 (200 ns) & $3.6 / 4.8$ & $1.4 / 3.0$ \\
\hline The model with fixed AM11542, Ser383 is mutated to Gly383 (200 ns) & $1.0 / 1.6$ & $3.2 / 4.7$ \\
\hline The model with free AM11542, Ser383 is mutated to Gly383 (200 ns) & $3.1 / 4.5$ & $1.4 / 2.3$ \\
\hline $\begin{array}{l}\text { The model with free AM11542, Leu359 and Met363 are mutated to } \\
\text { Gly359 and Gly363 (200 ns) }\end{array}$ & $1.6 / 2.4$ & $3.0 / 4.4$ \\
\hline The model with free AM11542, Phe379 is mutated to Gly379 (200 ns) & $3.0 / 4.5$ & $2.3 / 3.4$ \\
\hline
\end{tabular}

a The comparison for residues 351 - 361 of TM6 and 381 - 391 of TM7 (Figure 8).

b The "model" means the built model of inactive unliganded receptor, see text for further detail.

${ }^{\mathrm{c}}$ For all MD simulations, the structure averaged over the last $50 \mathrm{~ns}$ of trajectory is analyzed.

dFixation of ligand relative to fixed "basement".

e The location of the ligand's head was taken from results of $400 \mathrm{~ns}$ MD simulations of the model with free AM11542.

${ }^{\mathrm{f}}$ Usually a mutation to alanine is applied for mutagenesis studies. For every experiment with a mutation to glycine, the integrity of the $\alpha$-helix containing the mutated residue and stability of the $\alpha$-helix backbone was verified. 
One important consequence from the suggested explanation of the activation mechanism is that the geometry of the agonist tail link to the ligand head's core cannot be too flexible, as it expected to be highly strained at binding (red arrows in Figure 8). Indeed, in cores of numerous synthetic cannabinoid receptors agonists of all classes ${ }^{5}$ there are rigid unsaturated structures such as indoles and azaindoles which do not leave the tail's chain connection with such cores too much flexibility. Structural rigidity of, e.g., cannabinomimetic MDMB-fubinaca in its characteristic L-shape configuration could result in its high efficacy. ${ }^{8}$ One can also conclude that the flexible endocannabinoids could not serve as such a tensed connection between TM2 and the "basement" of TM3 and TM5 even if they were water soluble. Such lipid-soluble endocanabinoids should interact with $\mathrm{CB}_{1}$ in a quite different manner (see section Discussion and Conclusions).

In the following sections the receptor-agonist ligand interactions will be considered in detail and role of some important protein residues will be described.

\section{Free energy costs of the ligand tail and head bindings}

The "model of unliganded receptor" described above allows us to understand the role of ligand functional groups or particular protein residues in the receptor activation. If a change of ligand or a mutation of a residue affects the transformation of the receptor into its active state, one may suspect their important role in activation of the receptor.

In the activation mechanism discussed, the role of ligand's "tail" binding is crucial. Indeed, it is bound to the receptor in a narrow and deep hydrophobic pocket formed by side chains of Leu193, Thr197, Phe268, Ile271, Tyr275, Leu276, Trp279 and Met363. ${ }^{8}$ All of these residues, except for Met363, belong to the structural "basement" (TM3 and TM5 or the stiff loop between TM4 and TM5 reinforced by an S-S bridge). It is well known that neither the "tail" aliphatic chain nor its cyclic analogs (the fluorobenzyl one, for instance) cannot be too short or otherwise a substance ceases to be cannabinomimetic. ${ }^{5}$ If the chain is shorter than the propyl group, the binding of ligand notably decreases. ${ }^{39}$

To verify this dependence, we used ligand AM11542 from the crystal structure of the agonist$\mathrm{CB}_{1}$ complex. There were done several 200 ns scale $\mathrm{MD}$ simulations of the "model of unliganded receptor" immersed into membrane and included docked molecule of AM11542 with its tail shortened. All simulations with tail's aliphatic chain shorter than of five carbon atoms failed to result in an active conformation of the receptor (Table 2). For five carbon atoms and 
longer chains of the tail the final simulated structure was quite similar to results of simulations with AM11542 intact (Figures. 6 and 7).

The dependence of the binding free energy of AM11542 on the length of its aliphatic tail was studied by MD simulation technique using the original crystal structure ${ }^{6}$ of $\mathrm{CB}_{1}$-AM11542 complex. Details of those $\Delta \Delta \mathrm{G}$ calculations are given in section Methods. The values of $\Delta \Delta \mathrm{G}$ binding given in Table 3 suggest that the hydrophobic (i. e. mainly of entropic origin) free energies of the agonist tail binding are high enough. The free energy cost of the entire tail binding exceeds $14 \mathrm{kcal} / \mathrm{mol}$. When the tail is truncated to five carbon atoms chain, the binding free energy looses more than $5 \mathrm{kcal} / \mathrm{mol}$, but when it is truncated to four carbon atoms, the energy is reduced by a half. One can conclude that a loss of the tail binding energy of more than $7 \mathrm{kcal} / \mathrm{mol}$ might lead to impossibility to provide enough tightening force shown in Figure 8 to shift TM1, TM2, and TM7 in their active conformation placements and, as a result, to push TM6 out.

TABLE 3: $\Delta \Delta \mathrm{G}(\mathrm{kcal} / \mathrm{mol})$ at binding AM11542 with truncated tail: $\Delta \Delta \mathrm{G}=\Delta \mathrm{G}_{2}-\Delta \mathrm{G}_{1}$

\begin{tabular}{|c|c|c|c|c|c|}
\hline $\begin{array}{c}\text { Number of tail } \\
\text { carbon atoms }\end{array}$ & 0 & 3 & 4 & 5 & $8(\mathrm{Br})$ \\
\hline 0 & & & & & \\
\hline 3 & 6.22 & & -1.27 & & \\
\hline 4 & & & & & \\
\hline 5 & & 3.13 & 2.15 & & -5.40 \\
\hline $8(\mathrm{Br})$ & 14.31 & $\begin{array}{l}8.43 \\
8.29\end{array}$ & & & \\
\hline
\end{tabular}

The change of the binding free energy at thermodynamic transformation of entire ligand AM11542 into its tail without the ligand's head gives us energy of the head binding to the "hydrophobic lock". Such was done for the crystal structure of $\mathrm{CB}_{1}$ complex with AM11542 (section Methods, alchemical free energy calculations). The binding energies appeared to be even higher than those in the case of the tail interactions. The $\Delta \Delta \mathrm{G}$ binding energy exceeds 17 $\mathrm{kcal} / \mathrm{mol}$ for the entire planar structure of the head subunit of AM11542 (Table 1). From this value, the two outer cyclic structures of the head (the third one is linked to the tail) take about 10 $\mathrm{kcal} / \mathrm{mol}$ (the $\Delta \Delta \mathrm{G}$ difference between the whole and truncated heads). Thus, one can see that, according to the crystallographic structure of the complex with agonist ligand, the ligand's head 
is "glued" to TM2 even better than the tail to its hydrophobic pocket. In both cases the energy of hydrophobic interactions is high (from 14 to $17 \mathrm{kcal} / \mathrm{mol}$ ).

The simulated binding energies of the tail and head are close to each other (14 and 17 $\mathrm{kcal} / \mathrm{mol}$, respectively) - in fact they differ almost within accuracy of $\Delta \Delta \mathrm{G}$ simulations. It indicates the equality of mean forces acting on ligand's head and tail in opposite directions. Moreover, the binding energy is spent to the transformation of the receptor to its active-state conformation, not to the tension of the bonds at contacts of atomic groups of the protein and ligand. (The difference of the binding free energies of the head and tail will be spent on such local tension.) It directly follows from our previously mentioned experiment when we fixed the ligand head by a restraining potential and the ligand had no tail. The basement was spatially fixed in respect to the ligand head as well. MD simulations for such ligand fixation were done for our "unliganded-receptor" conformation. When we fixed position of the ligand head taken after docking calculations, an incomplete transformation of the receptor into its active-state structure was observed. But when we took a bit corrected location of the fixed ligand's head that followed from MD simulations of the model with free whole ligand (ligand positions difference is less than $1 \AA$ ), the transformation of the receptor into its active-state conformation by interactions with only such a fixed ligand's head was undistinguished from results of simulations with the whole ligand. (Table 2: "model with fixed AM11542 without its tail" and "model with precisely fixed AM11542 without its tail".) Now the energy of the tail interactions was accepted by the restraining potential (no visible shift) that provided the "tail" force marked in red in Figure 8, whereas the "head" force remained. No special interactions of protein residues with the ligand's tail in addition to its retaining in the binding pocket was needed to transform the protein into its active site - the tail was removed - only forces acting on the head in its final binding position fixed in respect to the basement were enough to do this. And even a slight incorrectness of the fixed head position led to a loss of the head binding energy on the local deformation of contacting atomic groups, not to the global structural rearrangement of the receptor.

\section{Energy of "toggle twin switch"}

Alpha helix TM6 that allosterically modulates binding of $\mathrm{G}$ protein to $\mathrm{CB}_{1}$ contains $\operatorname{Trp} 356$ the so called "toggle switch". This residue is conservative for GPCR receptors. Previous studies applying molecular simulations have confirmed its important role in activation of e.g. rhodopsin, ${ }^{48-49} \beta_{2}$-adrenergic receptor, ${ }^{50-51} \mathrm{M} 2$ muscarinic acetylcholine receptor ${ }^{52}$ or opioid receptor. ${ }^{53-54}$ In refs 8 and 9 the role of Trp356 is discussed. Especially it concerns its stacking 
interactions with Phe200 in the reported crystal structure of $\mathrm{CB}_{1}$ where the receptor is in its inactive state with antagonist ligand bound. The latter "twin toggle switch" interaction was assumed to play an important role in interaction with the antagonist ligands as well as in fixation of the inactive receptor structure. That conclusion is justified by the fact that mutations of Phe200 affect binding activity of $\mathrm{CB}_{1}{ }^{55}$

Our "unliganded-receptor model" is sensitive enough to be transformed into an active conformation at agonist ligand binding. It allows us to explore the role this "twin toggle switch" in the receptor activation. As follows from MD simulations, when agonist ligand AM11542 was bound to the "unliganded-receptor model" with mutated Trp356 or Phe200 to glycine, no transformation into the active state of receptor occurred and the receptor conformation remained intact (Table 2). But, on the other hand, the statistical analysis of trajectories suggests that conformation of residue $\mathrm{F} 200$ is rather unstable in this conserved inactive conformation of $\mathrm{CB}_{1}$ for the model studied. The latter facts rather suggest that preventing or impeding the receptor's structural transformation by these mutations cannot be explained by direct stacking interactions between Trp 356 and Phe 200, although such interaction takes place in the inactive structure of $\mathrm{CB}_{1}$.

To resolve this question, there were done MD simulations of the free energy perturbation to mutate both Trp356 and Phe200 into alanine residue. Results of the free energy changes $\Delta \mathrm{G}$ are collected in Table 4. The crystal structure of inactive $\mathrm{CB}_{1}$ was used (PDB ID: 5TGZ) with ligand removed.

TABLE 4: $\Delta \mathrm{G}$ of residue transformation $(\mathrm{kcal} / \mathrm{mol})$. Transformations of one residue in the presence of other residue.

\begin{tabular}{|c|c|c|c|}
\hline 356 & Ala & $\longrightarrow$ & Phe \\
\hline Ala & & -2.45 & \\
\hline$\downarrow$ & -6.94 & & -6.97 \\
\hline Trp & & -3.12 & \\
\hline
\end{tabular}

As seen from Table 4, the replacement of the side chain of Ala356 by Trp356 decreases the free energy by $6.94 \mathrm{kcal} / \mathrm{mol}$ in the absence of Phe200 side chain (Ala200) and by $6.97 \mathrm{kcal} / \mathrm{mol}$ in its presence. Thus, appearance of the side chain of Phe 200 gives only $-0.03 \mathrm{kcal} / \mathrm{mol}$. The difference in appearance of the side chain of Phe 200 caused by the presence of the Trp356 side chain is $-3.12-(-2.45)=-0.67 \mathrm{kcal} / \mathrm{mol}$. The difference between the two $\Delta \Delta \mathrm{G}$ values obtained (0.03 and -0.67) reflects accuracy of the simulations, as these values should be equal to each other. Anyway, we can see that the free energy of the stacking interactions between side chains 
of Phe200 and Trp356 in the protein environment is low: it is less than $0.7 \mathrm{kcal} / \mathrm{mol}$. Thus, the main contribution to a decrease of the free energy at appearance of the toggle switch Trp356 side chain is due to the entropic cost of the filling the cavity $(\sim 7 \mathrm{kCal} / \mathrm{mol})$, not due to weak stacking interactions with Phe200 (less than $1 \mathrm{kcal} / \mathrm{mol}$ ).

The negligible role of Phe200 in the inactive-state conformation also follows from MD simulations. As described above, after agonist ligand docking, $\mathrm{MD}$ simulations of $\mathrm{CB}_{1}$ in the "unliganded-receptor" conformation resulted in the active-state conformation of the receptor. Then we removed the ligand molecule and repeated the "inverse" simulations, but Phe200 was mutated to Gly200. The initial inactive-state conformation of the "empty receptor" was restored in a $200 \mathrm{~ns}$ MD run!

Anyway, the mutations of the "toggle twin switch" residues can prevent or impede activation of the receptor at agonist ligand binding. ${ }^{55}$ At least, it is confirmed in our experiments with the "unliganded model" with AM11542 docked (Table 2). When mutated to glycine, Gly356 or Gly200 does not allow the receptor's transformation into the active conformation for 200 ns run, whereas it takes place when Trp356 or Phe200 remains intact. But it suggests the significant increase of the free energy of the entire system due to expanding the void space due to the absence of the residue's side chain that could have happened if the outward movement of TM6 had taken place with such mutations. It would be purely entropic contribution, not the energy of stacking interactions between Trp356 and Phe200. Indeed, the entropic cost due to the absence of the side chains of the two residues is high enough: 7 and $3 \mathrm{kcal} / \mathrm{mol}$ (Table 4). When this emptiness expands, one could expect an increase of these values that might be notably higher than $\sim 0.7 \mathrm{kcal} / \mathrm{mol}$ of the 356-200 stacking interaction energy.

In a similar manner we observe an entropic contribution due to the neighboring locations of Trp356 and the ligand's tail. Indeed, as follows from Table 2, when the ligand is spatially fixed by the restraining potential, a partial activation of the receptor takes place when Trp356 is mutated to glycine. On comparing the two structures of the complexes averaged over the last 50 ns of MD simulations (Trp356 is mutated to glycine, ligand is free to move versus ligand is fixed), it is seen that in the case of free ligand, the ligand is shifted towards TM2 "hydrophobic lock". To some extent, it looks like the picture when the free-ligand tail is cut off. I. e., now the TM2-ligand interaction energy is spent on a deformation of the hydrophobic lock residues, not to the transformation of the entire 7TM domain because of the weaker ligand tail binding (see Figure 8). Thus, Trp356 "interacts" with the neighboring ligand, but rather in terms of the entropic contribution of filling voids into the free energy cost.

The interaction of Phe200 with the tail of agonist ligand is negligible. We do not mean the free energy of interaction of the side chain of Phe 200 with the hydrophobic pocket of the tail - it 
might be notable enough. The difference of Phe 200 interaction energies is considered when the void pocket is replaced by the pocket containing atoms of the ligand's tail. First, it follows from our experiment with the tail removal together with the ligand head fixation that there is no difference of MD simulations results for the receptor-ligand complex with free whole ligand and with only its fixed head (see above). Second, it follows from direct $\Delta \Delta \mathrm{G}$ simulations at mutation of Phe200 into Ala200. The thermodynamic transformations of the residue were done in the presence of the ligand and in its absence. The difference was within the statistics error (less than $0.5 \mathrm{kcal} / \mathrm{mol})$. The role of Phe 200 seems to be different: it could prevent penetration of ligands into the channel, providing their binding in the orthosteric binding pocket.

\section{Role of Phe379 and Ser383}

As mentioned above, the hydrophobic pocket of the ligand tail binding includes residues Trp279, Tyr275, Leu276, Ile271, Phe268, Met363, Leu193 and Thr197. The "hydrophobic lock" of TM2 interacting with the head of antagonist ligand includes residues Phe170, Phe174, Phe177, and His178. From the outer part of the channel, the ligand's head can also interact with residues Leu193, Phe268, Phe379, Leu359, Ser383, and Phe189. All of them were mentioned in

the earlier studies. ${ }^{8-9}$ Here we should emphasize an important role of residue Phe379 on the receptor activation, which has not been described before.

As shown in Figure 10, this residue not merely interacts with the ligand head, but it also noticeable changes its location in the final active structure of $\mathrm{CB}_{1}$. As follows from $\mathrm{MD}$ simulations, a mutation of this residue to one with a smaller side chain prevents from transformation of receptor into its active-state conformation (Table 2). The simplest explanation is a stronger attraction of TM7 due to free-energy entropic contribution of the released empty space. As a result, TM7 cannot easily move towards the "basement", as shown in Figure 7. Thus, this interaction of the side chain of Phe379 with the ligand head facilitates the movement of TM7 towards TM5, increasing pressing of TM7 on TM6.

Ser383 forms a hydrogen bond with the ligand's head. This bond is present for all agonist ligands (Table 1, Figure 1), as their polar oxygen atoms or hydroxyl groups are always found in close proximity of the serine residue hydroxyl group after ligand docking calculations. It could indicate the crucial role of this bond in the activation mechanism. This role is revealed by means of two MD simulations with the "unliganded model" of $\mathrm{CB}_{1}$ when Ser383 is mutated to glycine. At the first simulation AM11542 was fixed by a restraining potential. In the second case the docked agonist ligand is completely free. (The "basement" was fixed by restraining potential in both cases.) Results of RMSD comparison with active and inactive conformations of $\mathrm{CB}_{1}$ are 
presented in Table 2. We can see that in the case of fixed ligand the hydrogen bond energy itself is not crucial - results are undistinguished from simulations with fixed the ligand and "basement" for the "unliganded model" without any mutations. The final receptor conformation is close to its active-state conformation known from X-ray data. When the ligand was free to move, the mutation of Ser383 prevented from transformation of the receptor into its active-state structure. Moreover, the head of ligand moved close to the "hydrophobic lock" of TM2 residues Phe170, Phe174, and Phe177. There was notably shorter movement of the side chains of these residues towards the basement (TM3-TM5), instead the head of the ligand was distorted and attracted towards TM2. (The ligand's tail was found at the same position as it was from simulations of $\mathrm{CB}_{1}$ without the mutation.) In other words, the free energy of the ligand's head binding to the "hydrophobic lock" was spent not to the structural transformation of $\mathrm{CB}_{1}$ due to move of TM2 and TM7, but to the local deformation of the ligand at the contact of its head and the "hydrophobic lock" of TM2. I.e., the role of Ser383 is a stiff connection of the ligand's head to TM7 to transmit the ligand's interaction energy to the $\mathrm{CB}_{1}$ conformation rearrangement via movement of side chains of "hydrophobic lock" residues (Figure 8) and not just to the ligand's head deformation. This was clearly indicated in mutation experiments: Ser383 played a crucial role in mediating ligand specific interactions for agonists at $\mathrm{CB}_{1}{ }^{56}$

Two residues of the moving $\alpha$-helix TM6 directly interact with the agonist ligand: Leu359 and Met363. A mutation to both of them to glycine did not affect a capability of AM11542 to transform the inactive-state conformation of our "unliganded-receptor model" to an active state of the receptor on ligand binding (Table 2). Thus, these interactions should not be regarded as of importance for the receptor activation. 


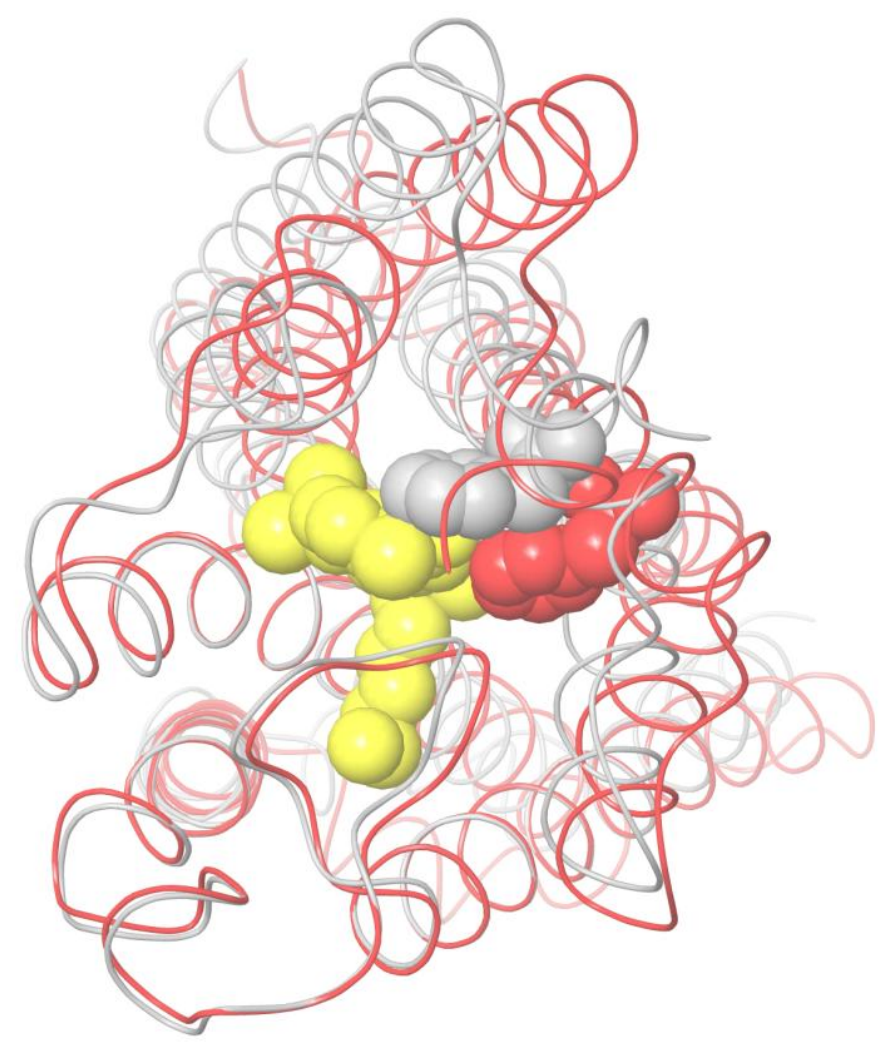

Figure 10. Interaction of Phe 379 with the head of ligand. The residue is shown in gray in the "unliganded-receptor model" and in red in the active conformation of the receptor interacting with agonist ligand (yellow).

\section{Simulations of $\mathrm{CB}_{1}$ activation with other agonist ligands}

The sensitivity of the "unliganded-receptor model" to be transformed into the active-state conformation of $\mathrm{CB}_{1}$ at exogenous agonist binding was verified for some other agonists from Table 1 and two ligands JWH-001 and JWH-002 that bind to $\mathrm{CB}_{1}$, look structurally similar to agonists, but they are not cannabimimetics (Figure 11). ${ }^{12}$ After docking into the "unligandedreceptor model" and a $50 \mathrm{~ns}$ equilibration MD run with the protein backbone atoms fixed, the protein was released (except for the "basement" backbone atoms) and a 200 ns MD simulation run of the receptor with ligand in the membrane environment was carried out. For some ligands the protein was transformed into its active-state conformation, but for some of them was not. For example, ligands JWH-018 and A-834735 led to the transforming into the active state such as was described above for ligand AM11542. It was reflected in the shifts of TM2, TM1, TM7 and, as a result, in allosteric modulating movement of TM6 as shown in Figure 7. Ligands JWH-001 and JWH-002 did not changed the conformation of TM2, TM6 and TM7. It was also expected, as they are not agonists of $\mathrm{CB}_{1}$. The receptor conformation remained intact too at $\mathrm{MD}$ simulations with endogenous agonists forced to be docked into the orthosteric binding site (as shown in Figure 1). It could suggest that their binding site is different. ${ }^{9}$ For other agonists the 
conformational transformation was not achieved in our computational experiments for $200 \mathrm{~ns}$ MD simulation runs, although tendencies to the movements depicted in Figure 7 were observed for some of them.

The simplest explanation of this failure is insufficient simulation time. The real time for achievement of the conformation transformation is unknown - we limited our attempts by $400 \mathrm{~ns}$ runs. The reason of a longer time to reach the transformation can be explained for $\Delta^{9}$-THC which is structurally quite similar to AM11542 (Table 1). The difference is only in the ligand's tail: for $\Delta^{9}$-THC, its aliphatic chain is shorter by three heavy atoms and the two methyl groups at the second atom of the chain are absent. From Table 3 we see that such a shortening of the tail leads to a loss of more than $5 \mathrm{kcal} /$ mole of the tail energy binding. The cost of the binding energy for the two methyl groups was studied separately. The $\Delta \Delta \mathrm{G}$ free energy perturbation technique was applied to estimate this value. The tail of AM11542 was transformed into a bare aliphatic chain terminated by a bromine atom. The cost of removal of one of the methyl groups was calculated to be about $1 \mathrm{kcal} / \mathrm{mol}$. Elimination of two $\mathrm{CH}_{3}$ groups of the tail of AM11542 led to a loss in the tail binding energy exceeding $2 \mathrm{kcal} / \mathrm{mol}$. Thus, the entire decrease of the tail binding energy for $\Delta^{9}$-THC in comparison with AM11542 exceeds $7 \mathrm{kcal} / \mathrm{mol}$. Indeed, $\Delta^{9}-\mathrm{THC}$ is known to be much less psychoactive substance than synthetic cannabinomimetics, therefore this weaker tail binding might lead to lower activity according to the $\mathrm{CB}_{1}$ activation mechanism considered. We can suggest that the failure to quickly activate the modeled apo structure of $\mathrm{CB}_{1}$ for other agonists might originate from the different energies of binding of their heads to TM2 hydrophobic lock as well as of their tails to the tail hydrophobic pocket. But the detailed analysis of these binding energies for all of them is beyond the framework of the present study.

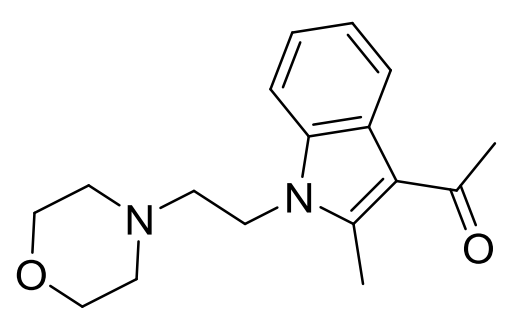

JWH-001

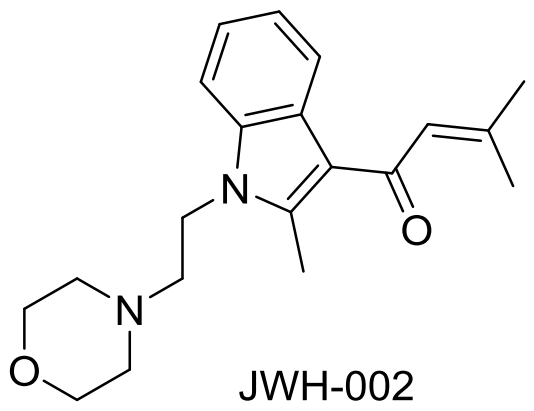

JWH-002

Figure 11. Ligands JWH-001 and JWH-002. 


\section{Discussion and Conclusions}

The suggested method of obtaining an artificial structural model of the unliganded 7TM domain of receptor $\mathrm{CB}_{1}$ allowed us to perform a transformation of the receptor to its active-state conformation via computer MD simulations of exogenous agonist binding to $\mathrm{CB}_{1}$. Above we quote the name of the model and call this model "chimeric" since it is not yet unknown conformation of this GPCR when there is no ligand in the orthosteric binding pocket. This model is just a metastable intermediate state between the known atomic structures of complexes of $\mathrm{CB}_{1}$ with antagonist and agonist ligands solved from crystallographic X-ray data. But the sensitivity of the model to ligand binding and a possibility of theoretical modeling of the receptor's active state have helped us to verify, confirm and complete the previously claimed features of the receptor activation mechanism. ${ }^{8,9}$

They can be summarized in the following main conclusions:

(i) Any (meta)stable conformation of the seven transmembrane $\alpha$-helices forms a shell around the solvent-accessible central channel. This quasi-cylindrical shell is quite resistant to changes of mutual locations of the adjoining helices and looks like a cylindrical shell made of very viscous and, at the same time, elastic substance.

(ii) The active-state structure of $\mathrm{CB}_{1}$ is unique for exogenous agonist ligands bound. It appears at maximal pressing on $\alpha$-helix TM6 between TM7 and TM5. This pressing is due to movements of TM2 and side chains of its residues in respect of solid structure of TM3, TM5 and TM4 and direct interactions of TM7 with the ligand bound in the orthosteric binding pocket. It finally presses TM6 out, forming the conformation of activated receptor. Moving TM6 outward is accompanied by the shift of the adjoining $\alpha$ helix TM7 towards $\alpha$-helix TM5, the opposite neighbor of TM6. This shift of TM7 takes place along the entire length of TM7 and TM6.

(iii) Binding of different inhibitors to the receptor does not lead to a single receptor's state they can presumably affect the receptor conformation in different ways to prevent appearance of the allosterically modulating conformation of TM6. Binding of known antagonists in the channel stretches the cylindrical shell leaving no possibility for $\alpha$-helix TM6 to protrude outside. On the contrary, binding of exogenous agonists of $\mathrm{CB}_{1}$ leads to macromolecule's internal tension shrinking the cylindrical shell.

(iv) There are three peculiar features of the agonist ligand structure (in addition to its size and flexibility to entry at the orthosteric binding pocket). The first one is high-energy stacking interactions of the ligand head with the "hydrophobic lock" of TM2 (residues F170, F174, F177, and H178). The second feature is high energy of hydrophobic interaction of the 
ligand's tail with residues of TM3 and TM5 in a deep and narrow hydrophobic pocket. The third one is a stiff connection between the ligand's tail and head: this link cannot be flexible. As a result, the ligand core connected to the tail has to be a rigid molecular structure. The latter is observed in reality for all known cannabinomimetics. And the tail has to be maximally long to strengthen its binding in the hydrophobic pocket. This conclusion agrees with properties of real cannabinomimetics as well. In fact, the core-tail link of the ligand plays the role of a bowstring between TM2 and TM7 and, on the other hand, TM3 and TM5. Attraction of TM2-TM7 and TM3-TM5 residues to the ligand molecule shrinks the channel shell along this bowstring, shifting TM1 and TM7 towards TM5 and finally pushing TM6 outward.

(v) The "toggle twin switch" Trp356-Phe200 interaction itself plays no role in retaining the inactive-state conformation of the receptor, although both residues are important for reaching its active state.

Some of these conclusions have been drawn in previously published researches, ${ }^{8-9}$ but others require comments. One conclusion concerns determining the "hydrophobic core" of the seventransmembrane domain of the receptor. According to our structural research, this core contains extracellular-side fragments of TM3, TM4, and TM5. They are involved in the surroundings of the agonist ligand orthosteric binding pocket. We called this rigid structure as "basement", as it interacts with $\mathrm{CB}_{1}$ ligand's tails. The core includes TM3 and TM4 (except, perhaps, the intracellular loop connecting them), and TM5 from its beginning to residue Leu286 after which TM5 gains flexibility at the structural activation change. The TM4-TM5 loop fixed by an S-S bond could also be added to this "basement". The nature of this core is revealed by comparisons of crystal complexes of $\mathrm{CB}_{1}$ with agonist and, on the other hand, with antagonist ligands bound. It is also clearly indicated in the thermal motion analysis via calculations of the backbone atoms' RMSD for MD runs of the receptor (Figure S2 in the Supporting Information). This hydrophobic region looks as mostly stable at the thermal motion - a usual definition of the hydrophobic core for proteins. The kink point or hinge of TM5 (Leu286) corresponds to proline in other class A GPCR proteins. ${ }^{57}$ This structural rigidity means a load in terms of the mean force. Indeed, instead of proline, in $\mathrm{CB}_{1}$ we find a strengthening of the rigid part of TM5 near that point by a hydrogen bond of the side chain of Ser284 with carboxyl oxygen of the Ile 280 (Figure 9B). The TM4 $\alpha$-helix also includes Pro251 in the region considered. The definition of the hydrophobic core explains the role of TM4 that is not directly involved into interactions with ligands - it forms the rigid "basement" of the molecular structure by numerous interactions with highly reinforced TM3. Finally, we draw the conclusion about a different method of superimposing active and inactive conformations of the receptor and a different indicator of its 
active state (Figure 8, Table 2). The use of TM6 movement as an indicator of the active state in the crystal complexes of GPCRs is unreliable. Coupling with the transducer such as Grotein seems to be necessary for GPCRs to adopt the fully active state. ${ }^{58}$ At crystallization transducers are replaced by G-protein mimetics such as e.g. camelid antibody fragments that bind directly to the intracellular side of GPCR, ${ }^{59-60}$ therefore the conformation of TM6 can be affected by this binding.

The ideas about activation of GPCRs were considered in recent reviews. ${ }^{59-62}$ Despite quite different sequences, agonist ligands and chemistry of their binding, the spatial backbone patterns of 7TM domains for class A GPCR (such as rhodopsin, $\beta$-adrenergic receptors or $\mu$-opioid receptor) can be superimposed on each other at low RMSD for both active and inactive conformations. It suggests a very similar mechanism of the entire structure transformation not completely understood yet. ${ }^{63}$ One of the described experimental results was a conclusion about "basal equilibrium" and multiple conformations of the unliganded receptor. ${ }^{64}$ Described switching between the inactive states within hundreds of microseconds and a high energy barrier to achieve the active state qualitatively agree with our conclusions from computer simulations of $\mathrm{CB}_{1}$. As described above, the free energy difference at rearrangement of the receptor topology is about $10 \mathrm{kcal} / \mathrm{mol}$ (according to the ligand's tail and head binding energies). (Here we assume the lower limit of the shortest tails binding energy, equality of the mean forces acting on the head and tail and total spending of this energy on the protein structural rearrangement, not on the local tension of interacting atomic groups.) But the energy barrier can be much higher than this final difference - this is the cause of the impossibility to reach the active state in simulations by just replacement of the inverse agonist by agonist ligand in the inactive structure. (It is why we built the intermediate unliganded model of still an inactive-state conformation where this barrier was significantly decreased.)

The limiting case of the active state observed for crystal complex of $\mathrm{CB}_{1}$ with agonist AM11542 (and achieved in our MD simulations for the "unliganded-receptor" conformation with AM11542 docked) does not contradict the fact that other ligand can cause other rearrangement of the 7TM domain structure when we deal with interacting of GPCR with different G-proteins or $\beta$-arrestin. ${ }^{62}$ It is merely stated that there is a definite structure rearrangement in a case of the full agonist (such as AM11542) corresponding to a GPCR-G $\mathrm{G}_{\mathrm{i}}$ complex. This activation is detected not by the outward movement of TM6 (which depends on the presence of nanobody or G-protein) ${ }^{65}$ but by the value of movement of TM7 towards TM5 (more than $3 \AA$ ) in a region shown in Figure 8 when we compare the active and inactive structures by means of superimposing the "hydrophobic cores" of the structures. Then a corresponding movement of the pressed out TM6 also should be about $3 \AA$ within this region. It 
can be illustrated for other class A GPCRs. In Figures S3, S4, and S5 (Supporting

Information) such a superposition is done for comparison of active and inactive $\mathrm{X}$-ray structures of $\beta_{2}$-adrenergic receptor, ${ }^{66-67} \mu$-opioid receptor, ${ }^{68-69}$ and rhodopsin, ${ }^{70-71}$ respectively. In all three cases one can see a picture quite similar to shown in Figure 8: TM7 and TM6 are moved by about $3 \AA$ towards TM5 in the region chosen for detection of the activation, i.e. in the fragments of TM6 and TM7 "above" TM5 taken up to the proline residue of TM5, where projections of TM7 and TM5 axes along the direction of the TM7 shift cross each other (Figure 9B). In all three cases TM3 is reinforced by two or three additional hydrogen bonds in a region similar to shown in Figure 9A.

In previous studies ${ }^{63,72}$ a similarity of conformation changes at class A GPCR activation is emphasized, although the changes are described differently. Despite the fact that the difference between that picture and our scheme is a matter of a choice of the "coordinate system" (i.e., what moves relative to what), the description of the "hydrophobic core" as an immovable part allows us to see the meaning of the receptor's structure transformation cleaner. In other works ${ }^{73}$ the suggested mechanism of the receptor activation is based entirely on the "molecular switches" buried in the receptor structure (such as the ionic lock between TM3 and TM6 fixing the inactive state). The role of these switches is undeniable, but they only support the main cause of the receptor transformation: the pressing out TM6 by the pushing TM7 towards TM5 of the "hydrophobic core" (Figure 8). The proof of a supplementary function of "molecular switches" was MD simulations of activation / inactivation of $\mathrm{CB}_{1}$ at insertion / removal of the agonist ligand to / from the orthosteric binding site, when this ionic lock was just removed (Table 2). In fact, the mechanism seems to be the same for all class A GPCRs, but the energy supply for the movement of TM7 towards TM5 differs according to different binding of different ligands. Another indirect suggestion of the pressing-TM6-out mechanism could be an experimentally observed shift to the active conformation of a GPCR under high pressure. ${ }^{74}$

The conclusion $(v)$ seems to contradict the experimentally established importance of Trp356 for the receptor activation. ${ }^{55}$ But Table 4 suggests not only a weak energy of Trp356-Phe200 stacking interaction (less than $1 \mathrm{kcal} / \mathrm{mol}$ ), but also a high energy of Trp356 mutation to alanine (about $7 \mathrm{kcal} / \mathrm{mol}$ ). Thus, the change of free energy at movement of TM6 can also differ noticeably in the presence of the side chain of Trp356 and in its absence. In other words, we should look not at the energy of Trp-Phe aromatic lock (the "twin toggle switch" energy), but at difference in the change of the free energy of the entire system at TM6 outward movement when Trp356 is replaced by Ala356.

In conclusions $(i)-(v)$ summarized above we see a simple molecular mechanism that has no crucial pair interactions (except, perhaps, with Ser383) and has only diffuse "hydrophobic 
gluing" of the ligand's head and tail to protein. It rather suggests that we deal not with evolution of the protein structure pointed to bind exogenous SCRAs, but with something that occasionally fit for some external compounds like $\Delta^{9}$-THC. Indeed, according to the discussed mechanism of receptor activation, molecules of the endogenous cannabinoids (which are apparently a result of evolution) cannot activate $\mathrm{CB}_{1}$ from inside the GPCR channel due to their flexibility. One can admit that such a structural feature as the deep pocket of the tail binding inside the channel might also appear occasionally. But it could hardly have happened since its emptiness had cost a significant increase of the protein free energy. Rather this hydrophobic pocket could serve some natural inhibitor binding.

\section{Supporting Information}

Figures S1-S5 and the CIF file of the atomic coordinates of the model of inactive apo receptor $\mathrm{CB}_{1}$.

\section{Acknowledgements}

The computational facilities of the First Moscow Medicine University are greatly acknowledged. The authors thank Dr. Stephan Ehrlich and Dr. Kai Welke (Schrodinger LLC) for valuable discussion. 


\section{References and Notes}

1. Marsicano, G.; Lutz, B. Expression of the Cannabinoid Receptor CB1 in Distinct Neuronal Subpopulations in the Adult Mouse Forebrain. Eur. J. Neurosci. 1999, 11, 4213-4225.

2. Herkenham, M.; Lynn, A. B.; Johnson, M. R.; Melvin, L. S.; De Costa, B. R.; Rice, K. C. Characterization and Localization of Cannabinoid Receptors in Rat Brain: a Quantitative in vitro Autoradiographic Study. J. Neurosci. 1991, 11, 563-583.

3. Szabo, B.; Schlicker, E. Effects of Cannabinoids on Neurotransmission. In Cannabinoids Ed. Pertwee R. G., Springer, Berlin, Heidelberg, 2005.

4. Mackie, K. Distribution of Cannabinoid Receptors in the Central and Peripheral Nervous System. In Cannabinoids. Ed. Pertwee R. G., Springer, Berlin, Heidelberg, 2005.

5. Banister, S. D.; Connor, M. The Chemistry and Pharmacology of Synthetic Cannabinoid Receptor Agonists as New Psychoactive Substances: Origins. In Handbook of Experimental Pharmacology, vol.252: New Psychoactive Substances. Ed. Maurer H. H. and Brandt S. D., Springer, 2018.

6. Hua, T.; Vemuri, K.; Nikas, S. P.; Laprairie, R. B.; Wu, Y.; Qu, L.; Pu, M.; Korde, A.; Jiang, S.; Ho, J.-H.; Han, G. W.; Ding, K.; Li, X.; Liu, H.; Hanson, M. A.; Zhao, S.; Bohn, L. M.; Makriyannis, A.; Stevens, R. C.; Liu, Z.-J. Crystal Structures of Agonist-Bound Human Cannabinoid Receptor $\mathrm{CB}_{1}$. Nature 2017, 547, 468-471.

7. Hua, T.; Vemuri, K.; Pu, M.; Qu, L.; Han, G. W.; Wu, Y.; Zhao, S.; Shui, W.; Li, S.; Korde, A.; Laprairie, R. B.; Stahl, E. L.; Ho, J.-H.; Zvonok, N.; Zhou, H.; Kufareva, I.; Wu, B.; Zhao, Q.; Hanson, M. A.; Bohn, L. M.; Makriyannis, A.; Stevens, R. C.; Liu, Z.-J. Crystal Structure of the Human Cannabinoid Receptor $\mathrm{CB}_{1}$. Cell 2016, 167, 750-762.

8. Kumar, K. K.; Shalev-Benami, M.; Robertson, M. J.; Hu, H.; Banister, S. D.; Hollingsworth, S. A.; Latorraca, N. R.; Kato, H. E.; Hilger, D.; Maeda, S.; Weis, W. I.; Farrens, D. L.; Dror, R. O.; Malhotra, S. V.; Kobilka, B. K.; Skiniotis, G. Structure of a Signaling Cannabinoid Receptor 1-G Protein Complex. Cell 2019, 176, 448-458.

9. Hua, T.; Li, X.; Wu, L.; Iliopoulos-Tsoutsouvas, C.; Wang, Y.; Wu, M.; Shen, L.; Johnston, C. A.; Nikas, S. P.; Song, F.; Song, X.; Yuan, S.; Sun, Q.; Wu, Y.; Jiang, S.; Grim, T. W.; Benchama, O.; Stahl, E. L.; Zvonok, N.; Zhao, S.; Bohn, L. M.; Makriyannis, A.; Liu, Z.-J. Activation and Signaling Mechanism Revealed by Cannabinoid Receptor- $\mathrm{G}_{i}$ Complex Structures. Cell 2020, 180, 655-665. 
10. Pertwee, R. G. The Diverse $\mathrm{CB}_{1}$ and $\mathrm{CB}_{2}$ Receptor Pharmacology of Three Plant Cannabinoids: $\Delta^{9}$-tetrahydrocannabinol, Cannabidiol and $\Delta^{9}$-tetrahydrocannabivarin. $B r . J$. Pharmacol. 2008, 153, 199-215.

11. Hollister, L. E. Structure-Activity Relationships in Man of Cannabis Constituents and Homologs and Metabolites of $\Delta^{9}$-tetrahydrocannabinol. Pharmacology 1974, 11, 3-11.

12. Wiley, J. L.; Marusich, J. A.; Huffman, J. W. Moving Around the Molecule: Relationship Between Chemical Structure and in vivo Activity of Synthetic Cannabinoids. Life Sci. 2014, 97, $55-63$.

13. Schrödinger Release 2017-1: Glide. Schrödinger, LLC, New York, NY, 2017.

14. Schrödinger Release 2017-1: LigPrep. Schrödinger, LLC, New York, NY, 2017.

15. UniProt Consortium. UniProt: a Worldwide Hub of Protein Knowledge. Nucleic Acids Res. 2019, 47(D1), D506-D515.

16. (a) Schrödinger Release 2017-1: Desmond Molecular Dynamics System. D. E. Shaw Research, New York, NY, 2017. (b) Schrödinger Release 2017-1: Maestro-Desmond Interoperability Tools. Schrödinger, New York, NY, 2017.

17. Harder, E.; Damm, W.; Maple, J.; Wu, C.; Reboul, M.; Xiang, J. Y.; Wang, L.; Lupyan, D.; Dahlgren, M. K.; Knight, J. L. ; Kaus, J. W.; Cerutti, D. S.; Krilov, G.; Jorgensen, W. L.; Abel, R.; Friesner, R. A. OPLS3: a Force Field Providing Broad Coverage of Drug-Like Small Molecules and Proteins. J. Chem. Theor. Comp. 2016, 12, 281-296.

18. (a) Schrödinger Release 2019-2: Desmond Molecular Dynamics System. D. E. Shaw Research, New York, NY, 2019. (b) Schrödinger Release 2019-2: Maestro-Desmond Interoperability Tools. Schrödinger, New York, NY, 2019.

19. Schrödinger Release 2019-2: FEP+. Schrödinger, LLC, New York, NY, 2019.

20. Abel, R.; Wang, L.; Harder, E. D.; Berne, B. J.; Friesner, R. A., Advancing Drug Discovery through Enhanced Free Energy Calculations, Acc. Chem. Res. 2017, 50, 1625-1632.

21. (a) Schrödinger Release 2019-2: Protein Preparation Wizard. Epik, Schrödinger, LLC, New York, NY, 2016; (b) Schrödinger Release 2019-2: Impact. Schrödinger, LLC, New York, NY, 2016; (c) Schrödinger Release 2019-2: Prime. Schrödinger, LLC, New York, NY, 2019.

22. Jacobson, M. P.; Friesner, R. A.; Xiang, Z.; Honig, B., On the Role of Crystal Packing Forces in Determining Protein Sidechain Conformations. J. Mol. Biol. 2002, 320, 597-608.

23. Roos, K.; Wu, C.; Damm, W.; Reboul, M.; Stevenson, J. M.; Lu, C.; Dahlgren, M. K.; Mondal, S.; Chen, W.; Wang, L.; Abel, R.; Friesner, R. A.; Harder, E. D. OPLS3e: Extending Force Field Coverage for Drug-Like Small Molecules. J. Chem. Theory Comput., 2019, 15, 1863-1874.

24. Schrödinger Release 2020-2: Glide, Schrödinger, LLC, New York, NY, 2020. 
25. Schrödinger Release 2020-2: LigPrep, Schrödinger, LLC, New York, NY, 2020.

26 . Lomize, M. A.; Pogozheva, I. D.; Joo, H.; Mosberg, H. I.; Lomize, A. L. OPM Database and PPM Web Server: Resources for Positioning of Proteins in Membranes. Nucleic Acids Res. 2012 40 (Database issue), D370-D376.

27. Wang, L.; Friesner, R. A.; Berne, B. J. Replica Exchange with Solute Scaling: A More Efficient Version of Replica Exchange with Solute Tempering (REST2). J. Phys. Chem. B 2011, $115,9431-9438$.

28. Ross, G. A.; Bodnarchuk, M. S.; Essex, J. W. Water Sites, Networks, and Free Energies with Grand Canonical Monte Carlo. J. Am. Chem. Soc. 2015, 137, 14930-14943.

29. Chin, C.-L.; Tovcimak, A. E.; Hradil, V. P.; Seifert, T. R.; Hollingsworth, P. R.; Chandran, P.; Zhu, C. Z.; Gauvin, D.; Pai, M.; Wetter, J.; Hsieh, G. C.; Honore, P.; Frost, J. M.; Dart, M. J.; Meyer, M. D.; Yao, B. B.; Cox, B. F.; Fox, G. B. Differential Effects of Cannabinoid Receptor Agonists on Regional Brain Activity Using Pharmacological MRI. Br. J. Pharmacol. 2008, 153, $367-379$.

30. Deng, H.; Gifford, A. N.; Zvonok, A. M.; Cui, G.; Li, X.; Fan, P.; Deschamps, J. R.; FlippenAnderson, J. L.; Gatley, S. J.; Makriyannis, A. Potent Cannabinergic Indole Analogues as Radioiodinatable Brain Imaging Agents for the $\mathrm{CB}_{1}$ Cannabinoid Receptor. J. Med. Chem. 2005, 48, 6386-6392.

31. Huffman, J. W.; Szklennik, P. V.; Almond, A.; Bushell, K.; Selley, D. E.; He, H.; Cassidy, M. P.; Wiley, J. L.; Martin, B. R. 1-Pentyl-3-phenylacetylindoles, a New Class of Cannabimimetic Indoles. Bioorg. Med. Chem. Lett. 2005, 15, 4110-4113.

32. Hillard, C. J.; Manna, S.; Greenberg, M. J.; DiCamelli, R.; Ross, R. A.; Stevenson, L. A.; Murphy, V.; Pertwee, R. G.; Campbell, W. B. Synthesis and Characterization of Potent and Selective Agonists of the Neuronal Cannabinoid Receptor $\left(\mathrm{CB}_{1}\right)$. J. Pharmacol. Exp. Ther. 1999, $289,1427-1433$.

33. Felder, C. C.; Joyce, K. E.; Briley, E. M.; Mansouri, J.; Mackie, K.; Blond, O.; Lai, Y.; Ma, A. L.; Mitchell, R. L. Comparison of the Pharmacology and Signal Transduction of the Human Cannabinoid $\mathrm{CB}_{1}$ and $\mathrm{CB}_{2}$ Receptors. Mol. Pharmacol. 1995, 48, 443-450.

34. Mechoulam, R.; Ben-Shabat, S.; Hanus, L.; Ligumsky, M.; Kaminski, N. E.; Schatz, A. R.; Gopher, A.; Almog, S.; Martin, B. R.; Compton, D. R.; Pertwee, R. G.; Griffin, G.; Bayewitch, M.; Barg, J.; Vogel, Z. Identification of an Endogenous 2-Monoglyceride, Present in Canine Gut, that Binds to Cannabinoid Receptors. Biochemical Pharmacology 1995, 50, 83-90.

35. Melvin, L. S.; Milne, G. M.; Johnson, M. R.; Subramaniam, B.; Wilken, G. H.; Howlett, A. C. Structure-Activity Relationships for Cannabinoid Receptor-Binding and Analgesic Activity: Studies of Bicyclic Cannabinoid Analogs. Mol. Pharmacol. 1993, 44, 1008-1015. 
36. Compton, D. R.; Johnson, M. R.; Melvin, L. S. and Martin, B. R. Pharmacological Profile of

a Series of Bicyclic Cannabinoid Analogs: Classification as Cannabimimetic Agents. J. Pharmacol. Exp. Ther. 1992, 260, 201-209.

37. Weissman, A.; Milne, G. M.; Melvin Jr, L. S. Cannabimimetic Activity from CP-47,497, a Derivative of 3-Phenylcyclohexanol. J. Pharmacol. Exp. Ther. 1982, 223, 516-523.

38. Melvin, L. S.; Milne, G. M.; Johnson, M. R.; Wilken, G. H.; Howlett, A. C. StructureActivity Relationships Defining the ACD-Tricyclic Cannabinoids: Cannabinoid Receptor Binding and Analgesic Activity. Drug. Des. Discov. 1995, 13, 155-166.

39. Aung, M. M.; Griffin, G.; Huffman, J. W.; Wu, M.; Keel, C.; Yang, B.; Showalter, V. M.; Abood, M. E.; Martin, B. R. Influence of the N-1 Alkyl Chain Length of Cannabimimetic Indoles upon CB(1) and CB(2) Receptor Binding. Drug Alcohol Depend. 2000, 60, 133-140.

40. Wiley, J. L.; Compton, D. R.; Dai, D.; Lainton, J. A.; Phillips, M.; Huffman, J. W.; Martin, B. R. Structure-Activity Relationships of Indole- and Pyrrole-Derived Cannabinoids. J. Pharmacol. Exp. Ther. 1998, 285, 995-1004.

41. Pryce, G.; Baker, D. Antidote to Cannabinoid Intoxication: the $\mathrm{CB}_{1}$ Receptor Inverse Agonist, $\mathrm{AM}_{251}$, Reverses Hypothermic Effects of the $\mathrm{CB}_{1}$ Receptor Agonist, CB-13, in Mice. Br. J. Pharmacol. 2017, 174, 3790-3794.

42. Gatley, S. J.; Lan, R.; Pyatt, B.; Gifford, A. N.; Volkow, N. D.; Makriyannis, A. Binding of the Non-Classical Cannabinoid $\mathrm{CP}_{55,490}$, and the Diarylpyrazole $\mathrm{AM}_{251}$ to Rodent Brain Cannabinoid Receptors. Life Sci. 1997, 61, PL 191-197.

43. Lan, R.; Lu, Q.; Fan, P.; Gatley, J.; Volkow, N. D.; Fernando, S. R.; Pertwee, R.; Makriyannis, A. Design and Synthesis of the CB1 Selective Cannabinoid Antagonist AM281: a Potential Human SPECT Ligand. AAPS PharmSci. 1999, 1, 39-45.

44. Fong, T. M.; Shearman, L. P.; Stribling, D. S.; Shu, J.; Lao, J.; Huang, C. R.-R.; Xiao, J. C.; Shen, C.-P.; Tyszkiewicz, J.; Strack, A. M.; DeMaula, C.; Hubert, M.-F.; GalijatovicIdrizbegovic, A.; Owen, R.; Huber, A. C.; Lanning, C. L. Pharmacological Efficacy and Safety Profile of Taranabant in Preclinical Species. Drug Dev. Res. 2009, 70, 349-362.

45. Griffith, D. A.; Hadcock, J. R.; Black, S. C.; Iredale, P. A.; Carpino, P. A.; DaSilva-Jardine, P.; Day, R.; DiBrino, J.; Dow, R. L.; Landis, M. S.; O'Connor, R. E.; Scott, D. O. Discovery of 1-[9-(4-chlorophenyl)-8-(2-chlorophenyl)-9H-purin-6-yl]-4-ethylaminopiperidine-4-carboxylic Acid Amide Hydrochloride (CP-945,598), a Novel, Potent, and Selective Cannabinoid Type 1 Receptor Antagonist. J. Med. Chem., 2009, 52, 234-237.

46. Shao, Z.; Yin, J.; Chapman, K.; Grzemska, M.; Clark, L.; Wang, J.; Rosenbaum, D. M. HighResolution Crystal Structure of the Human $\mathrm{CB}_{1}$ Cannabinoid Receptor. Nature 2016, 540, 602606. 
47. The described active structure of the receptor complex with AM11542 was reproduced in two other similar $200 \mathrm{~ns}$ MD runs at different seeds of random generator of starting atom velocities distribution.

48. Hornak, V.; Ahuja, S.; Eilers, M.; Goncalves, J. A.; Sheves, M.; Reeves, P. J.; Smith, S. O. Light Activation of Rhodopsin: Insights from Molecular Dynamics Simulations Guided by Solid-State NMR Distance Restraints. J. Mol Biol. 2010, 396, 510-527.

49. Takemoto, M.; Kato, H. E.; Koyama, M.; Ito, J.; Kamiya, M.; Hayashi, S.; Maturana, A. D.;

Deisseroth, K.; Ishitani, R.; Nureki, O. Molecular Dynamics of Channelrhodopsin at the Early Stages of Channel Opening. PLoS ONE 2015, 10, e0131094.

50. Shi, L.; Liapakis, G.; Xu, R.; Guarnieri, F.; Ballesteros, J. A.; Javitch, J. A. Beta2 Adrenergic Receptor Activation. Modulation of the Proline Kink in Transmembrane 6 by a Rotamer Toggle Switch. J. Biol. Chem. 2002, 277, 40989-40996.

51. Rosenbaum, D. M.; Rasmussen, S. G. F.; Kobilka, B. K. The Structure and Function of GProtein-Coupled Receptors. Nature 2009, 459, 356-363.

52. Dror, R. O.; Green, H. F.; Valant, C.; Borhani, D. W.; Valcourt, J. R.; Pan, A. C.; Arlow, D. H.; Canals, M.; Lane, J. R.; Rahmani, R.; Baell, J. B.; Sexton, P. M.; Christopoulos, A.; Shaw, D. E. Structural Basis for Modulation of a G-Protein-Coupled Receptor by Allosteric Drugs. Nature 2013, 503, 295-299.

53. Provasi, D.; Bortolato, A.; Filizola, M. Exploring Molecular Mechanisms of Ligand Recognition by Opioid Receptors with Metadynamics. Biochemistry 2009, 48, 10020-10029.

54. Shang, Y.; Yeatman, H. R.; Provasi, D.; Alt, A.; Christopoulos, A.; Canals, M.; Filizola, M. Proposed Mode of Binding and Action of Positive Allosteric Modulators at Opioid Receptors. ACS Chem. Biol. 2016, 11, 1220-1229.

55. McAllister, S. D.; Hurst, D. P.; Barnett-Norris, J.; Lynch, D.; Reggio, P. H.; Abood, M. E. Structural Mimicry in Class A G Protein-Coupled Receptor Rotamer Toggle Switches: the Importance of the F3.36(201)/W6.48(357) Interaction in Cannabinoid CB1 Receptor Activation. J. Biol. Chem. 2004, 279, 48024-48037.

56. Kapur, A.; Hurst, D. P.; Fleischer, D.; Whitnell, R.; Thakur, G. A.; Makriyannis, A.;

Reggio, P. H.; Abood, M. E. Mutation Studies of Ser7.39 and Ser2.60 in the Human CB $_{1}$ Cannabinoid Receptor: Evidence for a Serineinduced Bend in $\mathrm{CB}_{1}$ Transmembrane Helix 7. Mol. Pharmacol. 2007, 71, 1512-1524.

57. Elgeti, M.; Rose, A. S.; Bartl, F. J.; Hildebrand, P. W.; Hofmann, K.-P.; Heck, M. Precision vs Flexibility in GPCR Signaling. J. Am. Chem. Soc. 2013, 135, 12305-12312.

58. Nygaard, R.; Zou, Y.; Dror, R. O.; Mildorf, T. J.; Arlow, D. H.; Manglik, A.; Pan, A. C.; Liu, C. W.; Fung, J. J.; Bokoch, M. P.; Thian, F. S.; Kobilka, T. S.; Shaw, D. E.; Mueller, L.; Prosser, 
R. S.; Kobilka, B. K. The Dynamic Process of $\beta_{2}$-Adrenergic Receptor Activation. Cell 2013, $152,532-542$.

59. Venkatakrishnan, A. J.; Deupi, X.; Lebon, G.; Tate, C. G.; Schertler, G. F.; Babu, M. M. Molecular Signatures of G-Protein-Coupled Receptors. Nature 2013, 494, 185-194.

60. Erlandson, S. C.; McMahon, C.; Kruse, A. C. Structural Basis for G Protein-Coupled Receptor Signaling. Annu. Rev. Biophys. 2018, 47, 1-18.

61. Thal, D. M.; Glukhova, A.; Sexton, P. M.; Christopoulos, A. Structural Insights into GProtein-Coupled Receptor Allostery. Nature, 2018, 559, 45-53.

62. Seyedabadi, M.; Ghahremani, M. H.; Albert, P. R. Biased Signaling of G Protein Coupled Receptors (GPCRs): Molecular Determinants of GPCR/Transducer Selectivity and Therapeutic Potential. Pharmacol. Ther. 2019, 200, 148-178.

63. Zhou, Q.; Yang, D.; Wu, M.; Guo, Y.; Guo, W.; Zhong, L.; Cai, X.; Dai, A.; Jang, W.; Shakhnovich, E. I.; Liu, Z.-J.; Stevens, R. C.; Lambert, N. A.; Babu, M. M.; Wang, M.-W.; Zhao, S. Common Activation Mechanism of Class A GPCRs. eLife, 2019, 8, e50279. https://doi.org/10.7554/eLife.50279.

64. (a) Manglik, A.; Kim, T. H.; Masureel, M.; Altenbach, C.; Yang, Z.; Hilger, D.; Lerch, M. T.; Kobilka, T. S.; Thian, F. S.; Hubbell, W. L.; Prosser, R. S.; Kobilka, B. K. Structural Insights into the Dynamic Process of $\beta_{2}$-Adrenergic Receptor Signaling. Cell, 2015, 161, 1101-1111. (b) ibid 2015, 162, 1431.

65. Kim, T. H.; Chung, K. Y.; Manglik, A.; Hansen, A. L.; Dror, R. O.; Mildorf, T. J.; Shaw, D. E.; Kobilka, B. K.; Prosser, R. S. The Role of Ligands on the Equilibria Between Functional States of a G Protein-Coupled Receptor J. Am. Chem. Soc. 2013, 135, 9465-9474.

66. Ring, A. M.; Manglik, A.; Kruse, A. C.; Enos, M. D.; Weis, W. I.; Garcia, K. C.; Kobilka, B. K. Adrenaline-Activated Structure of Beta 2-Adrenoceptor Stabilized by an Engineered Nanobody. Nature 2013, 502, 575-579.

67. Warne, T.; Serrano-Vega, M. J.; Baker, J. G.; Moukhametzianov, R.; Edwards, P. C.; Henderson, R.; Leslie, A. G. W.; Tate, C. G.; Schertler, G. F. X. Structure of a $\beta_{1}$-Adrenergic GProtein-Coupled Receptor. Nature 2008, 454, 486-491.

68. Huang, W.; Manglik, A.; Venkatakrishnan, A. J.; Laeremans, T.; Feinberg, E. N.; Sanborn, A. L.; Kato, H. E.; Livingston, K. E.; Thorsen, T. S.; Kling, R. C.; Granier, S.; Gmeiner, P.; Husbands, S. M.; Traynor, J. R.; Weis, W. I.; Steyaert, J.; Dror, R. O.; Kobilka, B. K. Structural Insights into Mu-Opioid Receptor Activation. Nature 2015, 524, 315-321.

69. Manglik, A.; Kruse, A. C.; Kobilka, T. S.; Thian, F. S.; Mathiesen, J. M.; Sunahara, R. K.; Pardo, L.; Weis, W. I.; Kobilka, B. K.; Granier, S. Crystal Structure of the $\mu$-Opioid Receptor Bound to a Morphinan Antagonist. Nature 2012, 485, 321-326. 
70. Choe, H.-W.; Kim, Y. J.; Park, J. H.; Morizumi, T.; Pai, E. F.; Krauss, N.; Hofmann, K. P.; Scheerer, P.; Ernst, O. P. Crystal Structure of Metarhodopsin II. Nature 2011, 471, 651-655.

71. Palczewski, K.; Kumasaka, T.; Hori, T.; Behnke, C. A.; Motoshima, H.; Fox, B. A.; Le Trong, I.; Teller, D. C.; Okada, T.; Stenkamp, R. E.; Yamamoto, M.; Miyano, M. Crystal Structure of Rhodopsin: A G Protein-Coupled Receptor. Science 2000, 289, 739-745.

72. Tehan, B. G.; Bortolato, A.; Blaney, F. E.; Weir, M. P.; Mason, J. S. Unifying Family A GPCR Theories of Activation. Pharmacol. Ther. 2014, 143, 51-60.

73. Trzaskowski, B.; Latek, D.; Yuan, S.; Ghoshdastider, U.; Debinski, A.; Filipek, S. Action of Molecular Switches in GPCRs - Theoretical and Experimental Studies. Curr. Med. Chem. 2012, 19, 1090-1109.

74. Abiko, L. A.; Grahl, A.; Grzesiek, S. High Pressure Shifts the $\beta_{1}$-Adrenergic Receptor to the Active Conformation in the Absence of G Protein. J. Am. Chem. Soc. 2019, 141, 16663-16670. 